\title{
Axial kinetic theory and spin transport for fermions with arbitrary mass
}

\author{
Koichi Hattori, ${ }^{1}$ Yoshimasa Hidaka, ${ }^{2,3}$ and Di-Lun Yang ${ }^{1,4}$ \\ ${ }^{1}$ Yukawa Institute for Theoretical Physics, Kyoto University, Kyoto 606-8502, Japan \\ ${ }^{2}$ RIKEN Nishina Center, RIKEN, Wako, Saitama 351-0198, Japan \\ ${ }^{3}$ RIKEN Interdisciplinary Theoretical and Mathematical Sciences Program, RIKEN, Wako, \\ Saitama 351-0198, Japan \\ ${ }^{4}$ Faculty of Science and Technology, Keio University, Yokohama 223-8522, Japan
}

(Received 11 March 2019; revised manuscript received 25 September 2019; published 19 November 2019)

\begin{abstract}
We derive a quantum kinetic theory for fermions with arbitrary mass in a background electromagnetic field using a Wigner-function approach. Since spin of massive fermions is a dynamical degree of freedom (d.o.f.), kinetic equations with leading-order quantum corrections describe entangled dynamics of not only vector- and axial-charge distributions but also of the spin polarization. Therefore, we obtain one scalar and one axial-vector kinetic equations with magnetization currents pertinent to the spin-orbit interaction. We show that our results smoothly reduce to the massless limit where the spin of massless fermions is no longer an independent dynamical d.o.f. but is enslaved by the chirality and momentum, and the accordingly kinetic equations turn into chiral kinetic theory for Weyl fermions. We provide a kinetic theory covering both massive and massless cases and hence resolving the problem of constructing a bridge between them. Such a generalization may be crucial for applications to various physical systems. Based on our kinetic equations, we discuss the anomalous currents transported by massive fermions in thermal equilibrium.
\end{abstract}

DOI: 10.1103/PhysRevD.100.096011

\section{INTRODUCTION}

Triggered by predictions of the chiral magnetic effect (CME)/chiral vortical effect (CVE) [1-3], the transport of Weyl fermions has been widely studied in recent years. In light of connections to quantum anomalies, those transport phenomena have attracted much attention in systems with quite different energy scales, including relativistic heavy-ion collisions [4,5], Weyl semimetals [6], and lepton transport in supernova explosions $[7,8]$.

To investigate such anomalous transport in out-ofequilibrium systems, chiral kinetic theory (CKT) has been developed to capture the chiral anomaly effects [9-26]. Particularly, recent progress constructed a robust bridge between CKT and quantum field theory on the basis of the $\hbar$ expansion applied to the Wigner functions, which allows for systematic derivation of the side-jump effects stemming from spin-orbit coupling and collisions $[15,17,18]$.

However, the CKT developed for massless fermions appears to have an issue in its connection to the existing quantum kinetic theory for massive fermions [27-31]. There are crucial differences between the massless and massive

Published by the American Physical Society under the terms of the Creative Commons Attribution 4.0 International license. Further distribution of this work must maintain attribution to the author(s) and the published article's title, journal citation, and DOI. Funded by SCOAP . fermions as representations of the Lorentz symmetry. Whereas spin of Weyl fermion is enslaved by its momentum and is not an independent dynamical d.o.f., spin of massive Dirac fermions is subject to dynamical effects. It is thus necessary to understand how the side jumps and magneticmoment coupling in CKT are reduced from the dynamics of massive fermions to the massless limit.

In the aforementioned systems, mass effects will play sizable roles. For example, the measurements of global polarization for $\Lambda$ hyperons in heavy-ion collisions $[32,33]$ motivated by theoretical predictions [34,35] have triggered increasing studies of spin-polarization formation and angular momenta of relativistic fluids [36-43]. Since the spin of $\Lambda$ is attributed mainly to the strange-quark component, one may not treat them as massless fermions compared to the temperature of the quark-gluon plasma. In addition, the mass corrections upon the axial currents, generated by the axial CVE and the chiral separation effect (CSE), has accordingly received further attention [44-46]. As for the astrophysical applications of the chiral-plasma instability [47], a critical question was raised about the relaxation time of the axial charge due to the effects of electron mass $[48,49]$. These remain open questions and will be important applications of the CKT with the mass correction, which can simultaneously trace the time evolution of the charge transport, chiral imbalance, and spin polarization.

In this paper, we apply a Wigner-function approach to derive a quantum kinetic theory for fermions with arbitrary mass, which we call axial kinetic theory (AKT). Recently, 
related studies were presented in Refs. [50,51], in which the kinetic theories are derived in the rest frame of massive fermions. Although physics is frame invariant (analogous to gauge invariance), the choice of rest frame-similar to the choice of a particular gauge-is legitimate only for fermions with a mass larger than typical electromagnetic and gradient scales. Physically, one simply cannot define a rest frame for massless particles. The kinetic theories derived therein consequently causes divergence and the breakdown of $\hbar$ expansion for smaller masses. In order to apply a relativistic situation such as heavy-ion collisions, where the quark mass is smaller than or comparable to the gradient scale, one needs a theory applicable to an arbitrary frame (or at least a proper frame). AKT covers both the massive and massless cases and hence resolves the problem in constructing a bridge between them and should be regarded as an underlying theory which embodies the effective theories obtained in Refs. [50,51] for the largemass regime. After formulation, we discuss anomalous currents transported by massive fermions in a thermal equilibrium, which are important in heavy-ion collisions and neutron-star physics.

This paper is organized as follows. In Sec. II, we present master equations obtained from the Wigner-function approach. In Sec. III, the perturbative solution of the vector part of the Wigner functions (WFs) is derived, and a corresponding scalar kinetic equation in an axial-vector kinetic equation (AKE) is obtained. In Sec. IV, we further derive the axial part of the WFs partially with an alternative approach and generalization, where we also present a corresponding axial-vector kinetic equation in the AKE. In Sec. V, we discuss anomalous transport in thermal equilibrium in our formalism. We then give brief conclusions and an outlook in Sec. VI. Details of the derivations and computations are presented in the appendixes.

\section{WIGNER FUNCTIONS AND MASTER EQUATIONS}

We consider a massive Dirac field $\psi$, which is, unlike a massless Dirac field, no longer decomposed into a pair of Weyl fermions. The Wigner transformation applied to the quantum expectation values of the correlation functions reads

$$
S^{<(>)}(q, X)=\int d^{4} Y e^{\frac{i q \cdot Y}{\hbar}} S^{<(>)}(x, y),
$$

where $X=(x+y) / 2$ and $Y=x-y$. Here, $S^{<}(x, y)=$ $\langle\bar{\psi}(y) \psi(x)\rangle$ and $S^{>}(x, y)=\langle\psi(x) \bar{\psi}(y)\rangle$ are lesser and greater propagators, respectively. Hereafter, we focus on $S^{<}(x, y)$. Note also that the gauge link is implicitly embedded and $q^{\mu}$ thus represents the kinetic momentum. We then apply a decomposition based on the Clifford algebra [29],

$$
S^{<}=\mathcal{S}+i \mathcal{P} \gamma^{5}+\mathcal{V}^{\mu} \gamma_{\mu}+\mathcal{A}^{\mu} \gamma^{5} \gamma_{\mu}+\frac{\mathcal{S}^{\mu \nu}}{2} \Sigma_{\mu \nu}
$$

where $\Sigma_{\mu \nu}=i\left[\gamma_{\mu}, \gamma_{\nu}\right] / 2$ and $\gamma^{5}=i \gamma^{0} \gamma^{1} \gamma^{2} \gamma^{3}$. The coefficients $\mathcal{V}^{\mu}$ and $\mathcal{A}^{\mu}$ contribute to the vector- and axial-charge currents, while $\mathcal{S}$ and $\mathcal{P}$ are related to the quark and chiral condensates, respectively. The antisymmetric $\mathcal{S}^{\mu \nu}$ is related to magnetization.

For simplicity, we work in the regime where the collision effects are sufficiently weak and drop the contribution from the self-energy. Then the lesser propagator obeys

$$
(\text { (И }-m) S^{<}+\gamma^{\mu} i \frac{\hbar}{2} \nabla_{\mu} S^{<}=0,
$$

where $m$ is the mass of the fermion, $\nabla_{\mu}=\Delta_{\mu}+\mathcal{O}\left(\hbar^{2}\right)$, $\Pi_{\mu}=q_{\mu}+\frac{\hbar^{2}}{12}\left(\partial_{\rho} F_{\nu \mu}\right) \partial_{q}^{\rho} \partial_{q}^{\nu}+\mathcal{O}\left(\hbar^{4}\right)$, and $\Delta_{\mu}=\partial_{\mu}+F_{\nu \mu} \partial_{q}^{\nu}$, with $F_{\mu \nu}$ being the background-field strength. Equation (3) can be written into ten equations with 32 d.o.f. [29]. Three of them read

$$
\begin{aligned}
m \mathcal{S} & =\Pi \cdot \mathcal{V}, \quad m \mathcal{P}=-\frac{\hbar}{2} \nabla_{\mu} \mathcal{A}^{\mu}, \\
m \mathcal{S}_{\mu \nu} & =-\epsilon_{\mu \nu \rho \sigma} \Pi^{\rho} \mathcal{A}^{\sigma}+\frac{\hbar}{2} \nabla_{[\mu} \mathcal{V}_{\nu]},
\end{aligned}
$$

where $A_{[\mu} B_{\nu]} \equiv A_{\mu} B_{\nu}-B_{\nu} A_{\mu}$. Therefore, one can choose either eight functions $\mathcal{S}, \mathcal{P}$, and $\mathcal{S}^{\mu \nu}$ as a set of independent functions or the other half, $\mathcal{V}^{\mu}$ and $\mathcal{A}^{\mu}$ [52]. We choose the latter set and apply an $\hbar$ expansion to the rest of equations, which results in

$$
\Delta \cdot \mathcal{V}=0
$$

$$
\left(q^{2}-m^{2}\right) \mathcal{V}_{\mu}=-\hbar \tilde{F}_{\mu \nu} \mathcal{A}^{\nu}
$$

$$
q_{\nu} \mathcal{V}_{\mu}-q_{\mu} \mathcal{V}_{\nu}=\frac{\hbar}{2} \epsilon_{\mu \nu \rho \sigma} \Delta^{\rho} \mathcal{A}^{\sigma}
$$

$$
q \cdot \mathcal{A}=0
$$

$$
\begin{gathered}
\left(q^{2}-m^{2}\right) \mathcal{A}^{\mu}=\frac{\hbar}{2} \epsilon^{\mu \nu \rho \sigma} q_{\sigma} \Delta_{\nu} \mathcal{V}_{\rho}, \\
q \cdot \Delta \mathcal{A}^{\mu}+F^{\nu \mu} \mathcal{A}_{\nu}=\frac{\hbar}{2} \epsilon^{\mu \nu \rho \sigma}\left(\partial_{\sigma} F_{\beta \nu}\right) \partial_{q}^{\beta} \mathcal{V}_{\rho},
\end{gathered}
$$

where $\tilde{F}^{\mu \nu}=\epsilon^{\mu \nu \alpha \beta} F_{\alpha \beta} / 2$. We have retained the leadingorder quantum corrections and removed one redundant equation which can be reproduced from the above set; the detailed derivations are shown in Appendix A. 


\section{VECTOR WIGNER FUNCTIONS/SCALAR KINETIC EQUATION}

We now seek perturbative solutions $(\mathcal{V} / \mathcal{A})^{\mu}=$ $(\mathcal{V} / \mathcal{A})_{0}^{\mu}+\hbar(\mathcal{V} / \mathcal{A})_{1}^{\mu}$ up to $\mathcal{O}\left(\hbar^{1}\right)$. The zeroth-order solutions are immediately obtained from Eqs. (6)-(9) as

$$
\left(\mathcal{V}_{0} / \mathcal{A}_{0}\right)^{\mu}=2 \pi(q / a)^{\mu} \delta\left(q^{2}-m^{2}\right) f_{V / A},
$$

where $f_{V / A}(q, X)$ represent the vector/axial distribution functions. Here, $a^{\mu}(q, X)$ satisfies $q \cdot a=q^{2}-m^{2}$ and corresponds to the (non-normalized) spin four vector. As shown below, we have $a^{\mu}=q^{\mu}$ in the massless limit because the spin is enslaved by the momentum. However, $a^{\mu}$ is a dynamical variable in the massive case which should be determined by the kinetic theory.

Hence, we anticipate to derive the scalar kinetic equation (SKE) and axial-vector kinetic equation (AKE) governing the dynamical d.o.f. $f_{V / A}$ and $a^{\mu}$, Plugging Eq. (11) into Eqs. (5) and (7), one acquires the LO kinetic equations, $\delta\left(q^{2}-m^{2}\right) q \cdot \Delta f_{V}=0$ and $\delta\left(q^{2}-m^{2}\right) \square_{\mu \nu} \tilde{a}^{\nu}=0$, where $\tilde{a}^{\mu}=a^{\mu} f_{A}$ and $\square_{\mu \nu} \tilde{a}^{\nu}=q \cdot \Delta \tilde{a}_{\mu}+F_{\nu \mu} \tilde{a}^{\nu}$. The spin part is the renown Bargmann-Michel-Telegdi equation [53].

For $\mathcal{O}\left(\hbar^{1}\right)$ solutions, we first focus on the vector part, which can be derived from Eqs. (5)-(7). Similar to the massless case [17,22], Eqs. (6) and (7) determine the modification of the dispersion relation and the magnetization-current (MC) term, respectively. Accordingly, we find [54]

$$
\begin{gathered}
\mathcal{V}_{1}^{\mu}=2 \pi \tilde{F}^{\mu \nu} a_{\nu} \delta^{\prime}\left(q^{2}-m^{2}\right) f_{A}+2 \pi \delta\left(q^{2}-m^{2}\right) G^{\mu}, \\
G^{\mu}=\frac{\epsilon^{\mu \nu \rho \sigma} n_{\nu}}{2 q \cdot n}\left[\Delta_{\rho}\left(a_{\sigma} f_{A}\right)+F_{\rho \sigma} f_{A}\right],
\end{gathered}
$$

where $\delta^{\prime}\left(q^{2}-m^{2}\right) \equiv d \delta\left(q^{2}-m^{2}\right) / d q^{2}$, and $n^{\mu}(X)$ corresponds to a local frame vector specifying the spin basis. See Appendix B for more details of the derivation. The presence of MC term implies that $f_{V}$ is frame dependent, which follows the modified frame transformation between arbitrary frames $n^{\mu}$ and $n^{\prime \mu}$,

$$
f_{V}^{\left(n^{\prime}\right)}-f_{V}^{(n)}=\frac{\hbar \epsilon^{\lambda \nu \rho \sigma} n_{\lambda} n_{\nu}^{\prime}}{2(q \cdot n)\left(q \cdot n^{\prime}\right)}\left(\Delta_{\rho} a_{\sigma}+F_{\rho \sigma}\right) f_{A},
$$

as derived in Appendix F, where the superscripts $\left(n^{\prime}\right) /(n)$ of $f_{V}$ denote the frame dependence. Note that $f_{V / A}$ are frame independent at $\mathcal{O}\left(\hbar^{0}\right)$. When one defines the spin basis in the massive particle's rest frame, the explicit form of the frame vector reads $n^{\mu}=q^{\mu} / m$ such that $q \cdot n=m$, and the above expressions reduce to those obtained in Ref. [50], whereas this frame choice is only valid at large mass when $m S^{<} \gg\left|\gamma \cdot \Delta S^{<}\right|$. It is necessary to choose a different frame for smaller mass. See also Appendix E for further discussions upon this issue.

When $m=0$ and $a^{\mu}=q^{\mu}$, $G^{\mu}$ reproduces the side-jump term for massless fermions [15,17]. Inserting Eqs. (11)(13) into Eq. (5) yields the SKE up to $\mathcal{O}\left(\hbar^{1}\right)$,

$$
\begin{aligned}
0= & \delta\left(q^{2}-m^{2}\right)\left[q \cdot \Delta f_{V}+\hbar\left(\frac{E_{\mu} S_{a(n)}^{\mu \nu}}{q \cdot n} \Delta_{\nu}+S_{a(n)}^{\mu \nu}\left(\partial_{\mu} F_{\rho \nu}\right) \partial_{q}^{\rho}+\left(\partial_{\mu} S_{a(n)}^{\mu \nu}\right) \Delta_{\nu}\right) f_{A}\right]-\frac{\delta^{\prime}\left(q^{2}-m^{2}\right)}{q \cdot n} B^{\mu} \square_{\mu \nu} \tilde{a}^{\nu} \\
& +\frac{\hbar}{2} \delta\left(q^{2}-m^{2}\right) \epsilon^{\mu \nu \alpha \beta}\left[\Delta_{\mu}\left(\frac{n_{\beta}}{q \cdot n}\right)\left[\left(\Delta_{\nu} a_{\alpha}\right)+F_{\nu \alpha}\right]+\frac{n_{\beta}}{q \cdot n}\left(\left(\partial_{\mu} F_{\rho \nu}\right)\left(\partial_{q}^{\rho} a_{\alpha}\right)+\left[\left(\Delta_{\nu} a_{\alpha}\right)-F_{\rho \nu}\left(\partial_{q}^{\rho} a_{\alpha}\right)\right] \Delta_{\mu}\right)\right] f_{A},
\end{aligned}
$$

where $E_{\mu}=n^{\nu} F_{\mu \nu}, B^{\mu}=\frac{1}{2} \epsilon^{\mu \nu \alpha \beta} n_{\nu} F_{\alpha \beta}$, and

$$
S_{a(n)}^{\mu \nu}=\frac{\epsilon^{\mu \nu \alpha \beta} a_{\alpha} n_{\beta}}{2 q \cdot n}
$$

is the spin tensor. When $m=0$ and $a^{\mu}=q^{\mu}$, the second line in Eq. (15) vanishes and the first line reproduces the CKT in the massless case $[17,18,22]$. The detailed derivation of Eq. (15) is shown in Appendix D.

\section{AXIAL WIGNER FUNCTIONS/AXIAL-VECTOR KINETIC EQUATION}

The axial part of Wigner functions is obtained from Eqs. (8)-(10). However, unlike the vector part, Eqs. (8) and (9) lead only to the modified dispersion relation and do not uniquely fix the magnetization-current (MC) term. We thus obtain
$\mathcal{A}_{1}^{\mu}=2 \pi \tilde{F}^{\mu \nu} q_{\nu} \delta^{\prime}\left(q^{2}-m^{2}\right) f_{V}+2 \pi \delta\left(q^{2}-m^{2}\right) H^{\mu}$,

with an undetermined MC term $H^{\mu}$ up to the constraint $\delta\left(q^{2}-m^{2}\right) q \cdot H=0$ on shell. While Eq. (10) yields the AKE, we do not find any quantum correction when $H^{\mu}=0$ and $F_{\mu \nu}=0$.

In order to find the $\mathrm{MC}$ term for $\mathcal{A}_{1}^{\mu}$, we will implement an alternative method by constructing Wigner functions directly through the second quantization of free Dirac fields, as examined in the massless case [17]. The quantized free Dirac field reads [55]

$$
\psi(x)=\int \frac{d^{3} p}{(2 \pi)^{3}} \frac{1}{\sqrt{2 E_{\mathbf{p}}}} \sum_{s} u^{s}(p) e^{-i p \cdot x} a_{\mathbf{p}}^{s},
$$

where $E_{\mathbf{p}}=\sqrt{|\mathbf{p}|^{2}+m^{2}}$ and where we drop antifermions for simplicity. We have the annihilation (creation) operators $a_{\mathbf{p}}^{s(\dagger)}$ and the wave function $u^{s}(p)=\left(\sqrt{p \cdot \sigma} \xi^{s}, \sqrt{p \cdot \bar{\sigma}} \xi^{s}\right)^{T}$, 
with $s$ and $\xi^{s}$ being the spin indices and the two component spinor, respectively [55]. Here, $\sigma^{\mu}$ and $\bar{\sigma}^{\mu}$ are fourdimensional Pauli matrices which satisfy $\sigma^{\mu} \bar{\sigma}^{\nu}+\sigma^{\nu} \bar{\sigma}^{\mu}=$ $\bar{\sigma}^{\mu} \sigma^{\nu}+\bar{\sigma}^{\nu} \sigma^{\mu}=2 \eta^{\mu \nu}$, with the Minkowski matrix $\eta^{\mu \nu}$.

The lesser propagator then takes the form

$$
\begin{aligned}
& S^{<}(x, y)=\int \frac{d^{3} p}{(2 \pi)^{3}} \int \frac{d^{3} p^{\prime}}{(2 \pi)^{3}} \frac{1}{\sqrt{2 E_{\mathbf{p}}}} \frac{1}{\sqrt{2 E_{\mathbf{p}^{\prime}}}} \\
& \times \sum_{s, s^{\prime}} u^{s}(p) \bar{u}^{s^{\prime}}\left(p^{\prime}\right)\left\langle a_{\mathbf{p}^{\prime}}^{s^{\prime} \dagger} a_{\mathbf{p}}^{s}\right\rangle e^{i p_{-} \cdot X-i p_{+} \cdot Y},
\end{aligned}
$$

where $p_{+}^{\mu}=\left(p+p^{\prime}\right)^{\mu} / 2$ and $p_{-}^{\mu}=\left(p-p^{\prime}\right)^{\mu}$. The density operator can be written as $\left\langle a_{\mathbf{p}^{\prime}}^{s^{\prime} \dagger} a_{\mathbf{p}}^{s}\right\rangle=\delta_{s s^{\prime}} N_{V}\left(\mathbf{p}, \mathbf{p}^{\prime}\right)+$ $\mathcal{A}_{s s^{\prime}}\left(\mathbf{p}, \mathbf{p}^{\prime}\right)$, where $\mathcal{A}_{s s^{\prime}}\left(\mathbf{p}, \mathbf{p}^{\prime}\right) \neq 0$ when $s \neq s^{\prime}$. We parametrize the results of the spin sum as $\sum_{s} \xi_{s} \xi_{s}^{\dagger}=n \cdot \sigma=I$ and $\sum_{s, s^{\prime}} \xi_{s} \mathcal{A}_{s s^{\prime}} \xi_{s^{\prime}}^{\dagger}=S \cdot \sigma$ such that $S \cdot n=0$. After the Wigner transformation, we define

$$
\begin{gathered}
\tilde{f}_{V}(q, X) \equiv \int \frac{d^{3} p_{-}}{(2 \pi)^{3}} N_{V}\left(\boldsymbol{q}+\frac{\boldsymbol{p}_{-}}{2}, \boldsymbol{q}-\frac{\boldsymbol{p}_{-}}{2}\right) e^{-i p_{-} \cdot X}, \\
\hat{S}_{\mu}(q, X) \equiv \int \frac{d^{3} p_{-}}{(2 \pi)^{3}} S_{\mu}\left(\boldsymbol{q}+\frac{\boldsymbol{p}_{-}}{2}, \boldsymbol{q}-\frac{\boldsymbol{p}_{-}}{2}\right) e^{-i p_{-} \cdot X},
\end{gathered}
$$

where $\hat{S}_{\mu}(q, X)$ is related to the spin four vector. Further making the $\hbar$ expansion led by the $p_{-}^{\mu}$ expansion for wave functions analogous to the derivation in Ref. [17] for Weyl fermions, we find that Eq. (19) yields $(\mathcal{V} / \mathcal{A})^{\mu}$ in terms of $\tilde{f}_{V / A}$ with the explicit forms up to $\mathcal{O}\left(\hbar^{1}\right)$,

$$
\begin{array}{r}
\mathcal{V}^{\mu}=2 \pi \delta\left(q^{2}-m^{2}\right)\left[q^{\mu} f_{V}+\hbar \frac{\epsilon^{\mu \nu \alpha \beta} n_{\beta}}{2 q \cdot n} \partial_{\nu}\left(a_{\alpha} f_{A}\right)\right], \\
\mathcal{A}^{\mu}=2 \pi \delta\left(q^{2}-m^{2}\right)\left[a^{\mu} f_{A}+\hbar S_{m(n)}^{\mu \nu} \partial_{\nu} f_{V}\right] .
\end{array}
$$

Note that we have identified the present parametrizations to the previous ones as

$$
\begin{gathered}
f_{V}=\tilde{f}_{V}-\frac{\hbar S_{m(n)}^{\mu \nu}}{q \cdot n} \partial_{\nu} \hat{S}_{\mu}, \\
a \cdot n f_{A}=\hat{S} \cdot q, \quad a_{\perp \mu} f_{A}=\frac{(\hat{S} \cdot q) q_{\perp \mu}}{q \cdot n+m}-m \hat{S}_{\mu},
\end{gathered}
$$

where the subscripts " $\perp$ " denote the components perpendicular to $n^{\mu}$, i.e., $v_{\perp}^{\mu} \equiv v^{\mu}-(v \cdot n) n^{\mu}$ for the vector $v^{\mu}$. We also introduced the following tensor:

$$
S_{m(n)}^{\mu \nu}=\frac{\epsilon^{\mu \nu \alpha \beta} q_{\alpha} n_{\beta}}{2(q \cdot n+m)}=\frac{\epsilon^{\mu \nu \alpha \beta} q_{\alpha} n_{\beta}}{2 a \cdot n} .
$$

One may refer to Appendix $\mathrm{C}$ for details of the computations. The $\mathcal{V}_{1}^{\mu}$ in Eqs. (21) and (12) agree with each other when $F_{\mu \nu}=0$. Note that the previous constraint $q \cdot a=q^{2}-m^{2}$ is satisfied if we take $\hat{S} \cdot q=(q \cdot n+m) f_{A}$, which implies that $-\hat{S}^{\mu}=q_{\perp}^{\mu} f_{A} /(q \cdot n)$ when $m=0$. Thus, $(a \cdot n) /(2 q \cdot n)$ is identified with the helicity in the massless limit. From Eq. (24), one can obtain the second equality in Eq. (25).

In Eq. (22), one can read off the MC term [56]

$$
H^{\mu}=S_{m(n)}^{\mu \nu} \Delta_{\nu} f_{V}
$$

We generalize the derivative operator to include a background field analogous to the massless case [17], and we find that $\mathcal{A}^{\mu}$ has a symmetric form with $\mathcal{V}^{\mu}$ under interchanges $q^{\mu} \leftrightarrow a^{\mu}$ and $f_{V} \leftrightarrow f_{A}$. In Eq. (22), one could absorb $H^{\mu}$ by a redefinition $\bar{a}^{\mu} f_{A} \equiv a^{\mu} f_{A}+\hbar H^{\mu}$. The freedom of such a redefinition reveals itself as the nonuniqueness of the MC term, as we saw when solving the master equations (8) and (9) for $\mathcal{A}^{\mu}$, and it could occur in the massive case since $a^{\mu}$ is a dynamical variable to be determined by kinetic theory. However, it is crucial to explicitly separate the MC term $H^{\mu}$ from $a^{\mu}$ in order to see a smooth reduction to the CKT where $a^{\mu}$ is no longer an independent dynamical variable and is enslaved by $q^{\mu}$. The $H^{\mu}$ is also important for including the spin-orbit interaction. Note that $H^{\mu}=0$ when $n^{\mu}=q^{\mu} / m$, which is thus omitted in Refs. [50,51]. Similar to the case for $f_{V}, a^{\mu} f_{A}$ also obeys the following modified frame transformation,

$$
\begin{aligned}
& a^{\left(n^{\prime}\right) \mu} f_{A}^{\left(n^{\prime}\right)}-a^{(n) \mu} f_{A}^{(n)} \\
& \quad=\frac{\hbar \epsilon^{\mu \nu \alpha \beta}}{2}\left(\frac{n_{\beta}}{(q \cdot n+m)}-\frac{n_{\beta}^{\prime}}{\left(q \cdot n^{\prime}+m\right)}\right) q_{\alpha} \Delta_{\nu} f_{V} .
\end{aligned}
$$

Then, plugging Eqs. (11) and (17) into Eq. (10) and carrying out straightforward arrangements, we derive the AKE as

$$
\begin{aligned}
0= & \delta\left(q^{2}-m^{2}\right)\left(q \cdot \Delta\left(a^{\mu} f_{A}\right)+F^{\nu \mu} a_{\nu} f_{A}\right)+\hbar q^{\mu}\left\{\delta\left(q^{2}-m^{2}\right)\left[\left(\partial_{\alpha} S_{m(n)}^{\alpha \nu}\right) \Delta_{\nu}+\frac{S_{m(n)}^{\alpha \nu} E_{\alpha} \Delta_{\nu}}{q \cdot n+m}+S_{m(n)}^{\rho \nu}\left(\partial_{\rho} F_{\beta \nu}\right) \partial_{q}^{\beta}\right]\right. \\
& \left.-\delta^{\prime}\left(q^{2}-m^{2}\right) \frac{q \cdot B}{q \cdot n+m} q \cdot \Delta\right\} f_{V}+\hbar m\left\{\frac { \delta ( q ^ { 2 } - m ^ { 2 } ) \epsilon ^ { \mu \nu \alpha \beta } } { 2 ( q \cdot n + m ) } \left[m\left(\partial_{\alpha} n_{\beta}\right) \Delta_{\nu}+\left(m n_{\beta}+q_{\beta}\right)\left(\frac{\left(E_{\alpha}-\partial_{\alpha}(q \cdot n)\right)}{q \cdot n+m} \Delta_{\nu}\right.\right.\right. \\
& \left.\left.\left.-\left(\partial_{\nu} F_{\rho \alpha}\right) \partial_{q}^{\rho}\right)\right]+\delta^{\prime}\left(q^{2}-m^{2}\right) \frac{\left(m n_{\beta}+q_{\beta}\right) \tilde{F}^{\mu \beta}}{q \cdot n+m} q \cdot \Delta\right\} f_{V} .
\end{aligned}
$$


The detailed derivation is shown in Appendix D. Taking the massless limit $m \rightarrow 0$, one immediately finds that $a^{\mu}=q^{\mu}$ from Eq. (28) and that the full equation reduces to the CKT in Ref. [18] multiplied by $q^{\mu}$, which manifests the spin alignment along the momentum. In contrast, when $m \neq 0$, the background field and the derivative of the local frame vector engender nontrivial spin force.

When solving kinetic equations (15) and (28), we need to handle the terms proportional to $\delta^{\prime}\left(q^{2}-m^{2}\right)$. All of these terms can be arranged with the leading-order (LO) kinetic theory shown below Eq. (11): $2 \delta^{\prime}\left(q^{2}-m^{2}\right) q^{\mu} q \cdot \Delta f_{V}=$ $-\delta\left(q^{2}-m^{2}\right) \partial_{q}^{\mu}\left(q \cdot \Delta f_{V}\right)$ and $2 \delta^{\prime}\left(q^{2}-m^{2}\right) \square_{\mu \nu} \tilde{a}^{\nu}=-\delta\left(q^{2}-\right.$ $\left.m^{2}\right) \partial_{q}^{\rho}\left((q \cdot n)^{-1} n_{\rho} \square_{\mu \nu} \tilde{a}^{\nu}\right)$ up to $\mathcal{O}\left(\hbar^{1}\right)$. Then, all of the delta functions can be factored out from the CKTs.

From the solutions of the CKTs, one can get the vector/ axial currents and the symmetric/antisymmetric parts of the canonical energy-momentum tensor [57],

$$
J_{V / 5}^{\mu}=4 \int_{q}(\mathcal{V} / \mathcal{A})^{\mu}, \quad T_{S / A}^{\mu \nu}=2 \int_{q}\left(\mathcal{V}^{\mu} q^{\nu} \pm \mathcal{V}^{\nu} q^{\mu}\right),
$$

where $\int_{q} \equiv \int d^{4} q /(2 \pi)^{4}$. Angular-momentum conservation arises from Eq. (7) as discussed in the massless case [39], and $T_{A}^{\mu \nu}$ is responsible for the angular-momentum transfer (see Ref. [43] and the references therein). As an example, we consider the nonrelativistic limit with constant $n^{\mu}$ and $E^{\mu}$. By approximating $q^{\mu} \approx m n^{\mu}$, Eq. (28) yields $n \cdot \Delta\left(a^{\mu} f_{A}-\hbar \epsilon^{\mu \nu \alpha \beta} E_{\alpha} n_{\beta} \partial_{q \nu}\left(f_{V} / 4\right)\right) \approx 0$ after dropping the subleading terms in $m$ and arranging the delta functions with the aforementioned strategy. Then, we find a spin Hall current in the stationary state,

$$
J_{5}^{\mu} \approx-2 \pi \hbar \epsilon^{\mu \nu \alpha \beta} E_{\alpha} n_{\beta} \int_{q} \delta\left(q^{2}-m^{2}\right) \partial_{q \nu} f_{V} .
$$

\section{ANOMALOUS TRANSPORT IN THERMAL EQUILIBRIUM}

As an application, we discuss the mass effects on the anomalous transport in global equilibrium with constant thermal vorticity and chemical potentials, and we compare our conclusions from the SKE and AKE with those from the Kubo formula calculations.

While collisionless kinetic equations do not uniquely determine equilibrium WFs [18], we may construct equilibrium WFs motivated by the following considerations. For the vector charges, we may naturally take the Fermi distribution function $f_{V \mathrm{eq}}=f_{0}\left(q \cdot u-\mu_{V}\right)=1 /$ $\left(\exp \left(\beta\left(q \cdot u-\mu_{V}\right)\right)+1\right)$, where $\beta=1 / T$ and $\mu_{V}$ are the inverse temperature and vector chemical potential, respectively. On the other hand, the axial charge should be damped out as $t \rightarrow \infty$ when $m \neq 0$ because of the scattering. Thus, $f_{\text {Aeq }}$ may be at most $\mathcal{O}\left(\hbar^{1}\right)$ induced by the vorticity correction. Referring to the massless case [16,18], we also expect that $\mathcal{A}_{\mathrm{eq}}^{\mu}$ does not have an explicit dependence on $n^{\mu}$. Thus, we propose an equilibrium Wigner function in constant magnetic field and thermal vorticity

$$
\begin{gathered}
\mathcal{V}_{\mathrm{eq}}^{\mu}=2 \pi \delta\left(q^{2}-m^{2}\right) q^{\mu} f_{0}, \\
\mathcal{A}_{\mathrm{eq}}^{\mu}=2 \pi \hbar\left[\frac{\delta\left(q^{2}-m^{2}\right)}{4} q_{\nu} \epsilon^{\nu \mu \alpha \beta} \Omega_{\alpha \beta} \partial_{q \cdot \beta}\right. \\
\left.+\tilde{F}^{\mu \nu} q_{\nu} \delta^{\prime}\left(q^{2}-m^{2}\right)\right] f_{0},
\end{gathered}
$$

where $\Omega_{\mu \nu}=\partial_{[\mu}\left(\beta_{\nu]}\right) / 2$ corresponds to the thermal vorticity and $\beta_{\nu}=\beta u_{\nu}$. The equilibrium $\mathcal{A}_{\text {eq }}^{\mu}$ takes the equivalent form as the one for massless fermions at constant temperature except for the on-shell condition [18] and was also proposed for massive fermion [36] (a similar form to [37]), which satisfies the master equations. See Refs. [58,59] for WFs beyond weak vorticity and with acceleration.

The equilibrium Wigner functions (31) and (32) now lead to the CSE and the axial CVE, $J_{B / \omega 5}^{\mu}=\sigma_{B / \omega}(B / \omega)^{\mu}$, where

$$
\sigma_{B / \omega}=\frac{\hbar}{2 \pi^{2}} \int_{0}^{\infty} d|\mathbf{q}| g_{B / \omega} f_{0}^{(-/+)}\left(E_{\mathbf{q}}\right)
$$

with $g_{B}=1, g_{\omega}=\left(2 E_{\mathbf{q}}^{2}-m^{2}\right) / E_{\mathbf{q}}$, and $f_{0}^{( \pm)}=f_{0}\left(E_{\mathbf{q}}-\mu_{V}\right) \pm$ $f_{0}\left(E_{\mathbf{q}}+\mu_{V}\right)$. Here, the fluid vorticity $\omega^{\mu}$ is defined as $\omega^{\mu} \equiv T u_{\nu} \epsilon^{\mu \nu \alpha \beta} \Omega_{\alpha \beta} / 2=\epsilon^{\mu \nu \alpha \beta} u_{\nu}\left(\partial_{\alpha} u_{\beta}\right) / 2$. The above results agree with those derived from the Kubo formula with thermal correlators [45,46]. Similar to the massless case [15,16,18], a part of the axial CVE comes from the MC term which can be identified by comparing $\mathcal{A}_{\mathrm{eq}}^{\mu}$ with the general form (22).

Finally, since $f_{\text {Aeq }}=\mathcal{O}\left(\hbar^{1}\right)$, we conclude that the CME and vector CVE vanish at equilibrium when $m \neq 0$. On the other hand, it was shown by the thermal field theoretical calculation that the CME at equilibrium receives no mass correction $[60,61]$. However, the axial chemical potential $\mu_{5}$ is not a static quantity in the massive case, and the thermal field theory with a constant $\mu_{5}$ does not correctly capture its dynamics. The thermal field theoretical calculation may work only under certain caveats on the existence of $\mu_{5}$, and there are no equilibrium currents at strict thermal equilibrium at $\mu_{5}=0$. Only when equilibrium statistical operators breaking charge conjugation, parity, and rotation symmetry (e.g., with acceleration and chemical potential) exist would vector currents be allowed.

\section{CONCLUSIONS AND OUTLOOK}

In this work, we developed the quantum kinetic theory for arbitrary-mass fermions, which provides a theoretical framework for describing the coupled dynamics among the spin and the vector and axial charges. Moreover, we constructed a bridge on the long-standing gap between 
the CKT and axial kinetic theory. In the future, we will include collision effects to investigate their relaxation dynamics. It is feasible with an extension of the collision terms developed in the massless limit [17-19], and with deeper understandings and techniques obtained in this work.

\section{ACKNOWLEDGMENTS}

The authors thank Igor Shovkovy and Qun Wang for the fruitful discussions during the Workshop on Recent Developments in Chiral Matter and Topology, and XuGuang Huang, Yu-Chen Liu, and Kazuya Mameda when K. H. visited Fudan University. Y. H. was partially supported by the Japan Society for the Promotion of Science (JSPS), Grants-in-Aid for Scientific Research (KAKENHI) Grants No. 15H03652, No. 16K17716, and No. 17H06462. Y.H. was also partially supported by the RIKEN iTHES Project and the iTHEMS Program. K. H. and D.-L. Y. are supported in part by the Yukawa International Program for Quark-Hadron Sciences (YIPQS). D.-L. Y. is also partially supported by the Keio Institute of Pure and Applied Sciences (KiPAS) project in Keio University.

\section{APPENDIX A: DERIVATION OF MASTER EQUATIONS}

In this section, we derive the six master equations for the Wigner functions of Dirac fermions. We shall start with the Dirac Lagrangian density,

$$
\mathcal{L}=\bar{\psi}(i \not D-m) \psi,
$$

where the covariant derivative is $\mathcal{D}_{\mu}=\partial_{\mu}+i A_{\mu} / \hbar$ with the $\mathrm{U}(1)$ gauge field $A_{\mu}$. We define the greater and lesser propagators as

$$
\begin{aligned}
& {\left[S^{>}(x, y)\right]_{\alpha \beta} \equiv e^{\frac{i}{h} \int_{x}^{y} A_{\mu}(z) d z^{\mu}}\left\langle\psi_{\alpha}(x) \bar{\psi}_{\beta}(y)\right\rangle,} \\
& {\left[S^{<}(x, y)\right]_{\alpha \beta} \equiv e^{\frac{i}{\hbar} \int_{x}^{y} A_{\mu}(z) d z^{\mu}}\left\langle\bar{\psi}_{\beta}(y) \psi_{\alpha}(x)\right\rangle .}
\end{aligned}
$$

Here, $\alpha$ and $\beta$ denote the spinor indices.

After the Wigner transformation defined as

$$
S^{\lessgtr}(q, X)=\int \frac{d^{4} Y}{(2 \pi)^{4}} e^{\frac{i}{\hbar} q \cdot Y} S^{\lessgtr}(X+Y / 2, X-Y / 2),
$$

the lesser propagator without collision terms obeys

$$
\begin{gathered}
(\text { И }-m) S^{<}+\gamma^{\mu} i \frac{\hbar}{2} \nabla_{\mu} S^{<}=0, \\
S^{<}\left(\text {(И-m) }-i \frac{\hbar}{2} \nabla_{\mu} S^{<} \gamma^{\mu}=0,\right.
\end{gathered}
$$

or equivalently,

$$
\begin{aligned}
& \left\{\left(\text { (И-m), } S^{<}\right\}+\frac{i \hbar}{2}\left[\gamma^{\mu}, \nabla_{\mu} S^{<}\right]=0,\right. \\
& {\left[(\Pi-m), S^{<}\right]+\frac{i \hbar}{2}\left\{\gamma^{\mu}, \nabla_{\mu} S^{<}\right\}=0,}
\end{aligned}
$$

where we introduce

$$
\begin{aligned}
& \nabla_{\mu}=\partial_{\mu}+j_{0}(\square) F_{\nu \mu} \partial_{q}^{\nu}, \\
& \Pi_{\mu}=q_{\mu}+\frac{\hbar}{2} j_{1}(\square) F_{\nu \mu} \partial_{q}^{\nu}, \quad \square=\frac{\hbar}{2} \partial_{\rho} \partial_{q}^{\rho} .
\end{aligned}
$$

Here, we define $\partial_{\mu} \equiv \partial / \partial X^{\mu}, \partial_{q}^{\mu} \equiv \partial / \partial q_{\mu}$, and $F_{\mu \nu} \equiv$ $\partial_{\mu} A_{\nu}-\partial_{\nu} A_{\mu}$. Also, $j_{0}(\square), j_{1}(\square)$ are modified Bessel functions. We note that $\partial_{\rho}$ in $\square$ only acts on $F_{\nu \mu}$ when having spacetime-dependent background fields. Making the $\hbar$ expansion, which corresponds to the gradient expansion for $\partial_{\mu} \ll q_{\mu}$, one finds that

$\nabla_{\mu}=\partial_{\mu}+F_{\nu \mu} \partial_{q}^{\nu}-\frac{\hbar^{2}}{24}\left(\partial_{\rho} \partial_{\lambda} F_{\nu \mu}\right) \partial_{q}^{\lambda} \partial_{q}^{\rho} \partial_{q}^{\nu}+\mathcal{O}\left(\hbar^{4}\right)$,

$\Pi_{\mu}=q_{\mu}+\frac{\hbar^{2}}{12}\left(\partial_{\rho} F_{\nu \mu}\right) \partial_{q}^{\rho} \partial_{q}^{\nu}+\mathcal{O}\left(\hbar^{4}\right)$.

We then apply the decomposition based on the Clifford algebra,

$$
S^{<}=\mathcal{S}+i \mathcal{P} \gamma^{5}+\mathcal{V}_{\mu} \gamma^{\mu}+\mathcal{A}_{\mu} \gamma^{5} \gamma^{\mu}+\frac{\mathcal{S}_{\mu \nu}}{2} \Sigma^{\mu \nu},
$$

where $\Sigma^{\mu \nu}=i\left[\gamma^{\mu}, \gamma^{\nu}\right] / 2$ and $\gamma^{5}=i \gamma^{0} \gamma^{1} \gamma^{2} \gamma^{3}$.

For simplicity, we consider the collisionless case, in which Eq. (A6) results in

$$
\begin{gathered}
m \mathcal{V}_{\mu}=\Pi_{\mu} \mathcal{S}-\frac{\hbar}{2} \nabla^{\nu} \mathcal{S}_{\nu \mu}, \\
2 m \mathcal{A}^{\mu}=-\epsilon^{\mu \nu \rho \sigma} \Pi_{\sigma} \mathcal{S}_{\nu \rho}+\hbar \nabla^{\mu} \mathcal{P}, \\
m \mathcal{S}=\Pi \cdot \mathcal{V}, \\
m \mathcal{P}=-\frac{\hbar}{2} \nabla_{\mu} \mathcal{A}^{\mu}, \\
m \mathcal{S}_{\mu \nu}=-\epsilon_{\mu \nu \rho \sigma} \Pi^{\rho} \mathcal{A}^{\sigma}+\frac{\hbar}{2} \nabla_{[\mu} \mathcal{V}_{\nu]},
\end{gathered}
$$

and

$$
\begin{gathered}
\nabla^{\mu} \mathcal{V}_{\mu}=0, \\
\Pi \cdot \mathcal{A}=0, \\
\Pi_{\nu} \mathcal{V}_{\mu}-\Pi_{\mu} \mathcal{V}_{\nu}-\frac{1}{2} \epsilon_{\mu \nu \rho \sigma} \hbar \nabla^{\rho} \mathcal{A}^{\sigma}=0, \\
2 \Pi^{\nu} \mathcal{S}_{\nu \mu}+\hbar \nabla_{\mu} \mathcal{S}=0,
\end{gathered}
$$




$$
\epsilon^{\mu \nu \rho \sigma} \hbar \nabla_{\sigma} \mathcal{S}_{\nu \rho}+4 \Pi^{\mu} \mathcal{P}=0
$$

By writing $\mathcal{S}, \mathcal{P}$, and $\mathcal{S}_{\mu \nu}$ in terms of $\mathcal{V}_{\mu}$ and $\mathcal{A}_{\mu}$ from Eqs. (A12)-(A14), we obtain

$$
\nabla \cdot \mathcal{V}=0
$$

$\left(\Pi_{\mu} \Pi \cdot \mathcal{V}-m^{2} \mathcal{V}_{\mu}\right)=-\frac{\hbar}{2} \epsilon^{\nu \mu \rho \sigma} \nabla^{\nu} \Pi^{\rho} \mathcal{A}^{\sigma}+\frac{\hbar^{2}}{4} \nabla^{\nu} \nabla_{[\nu} \mathcal{V}_{\mu]}$

$$
\begin{gathered}
\Pi \cdot \mathcal{A}=0, \\
\Pi_{\nu} \mathcal{V}_{\mu}-\Pi_{\mu} \mathcal{V}_{\nu}=\frac{\hbar}{2} \epsilon_{\mu \nu \rho \sigma} \nabla^{\rho} \mathcal{A}^{\sigma}, \\
\left(\Pi^{2}-m^{2}\right) \mathcal{A}^{\mu}-\Pi_{\sigma} \Pi^{\mu} \mathcal{A}^{\sigma}=\frac{\hbar}{2} \epsilon^{\mu \nu \rho \sigma} \Pi_{\sigma} \nabla_{\nu} \mathcal{V}_{\rho}-\frac{\hbar^{2}}{4} \nabla^{\mu} \nabla \cdot \mathcal{A}, \\
\hbar\left(\left(\Pi^{\mu} \nabla_{\sigma}\right) \mathcal{A}^{\sigma}+\nabla_{\sigma} \Pi^{\sigma} \mathcal{A}^{\mu}\right)=\frac{\hbar^{2}}{4} \epsilon^{\mu \nu \rho \sigma} \nabla_{\sigma} \nabla_{[\nu} \mathcal{V}_{\rho]}, \quad(\mathrm{A} 23) \\
\hbar\left(\Pi \cdot \nabla \mathcal{V}_{\mu}+\left(\nabla_{\mu} \Pi^{\nu}\right) \mathcal{V}_{\nu}\right)=2 \epsilon_{\nu \mu \rho \sigma}\left(\Pi^{\nu} \Pi^{\rho}\right) \mathcal{A}^{\sigma}, \quad(\mathrm{A} 25)
\end{gathered}
$$

where $A_{[\mu} B_{\nu]} \equiv A_{\mu} B_{\nu}-B_{\nu} A_{\mu}$. Up to $\mathcal{O}(\hbar)$, Eqs. (A20)(A26) read

$$
\begin{gathered}
\Delta \cdot \mathcal{V}=0 \\
q_{\mu} q \cdot \mathcal{V}-m^{2} \mathcal{V}_{\mu}=-\hbar \tilde{F}_{\mu \sigma} \mathcal{A}^{\sigma}+\frac{\hbar}{2} \epsilon_{\mu \nu \rho \sigma} q^{\rho} \Delta^{\nu} \mathcal{A}^{\sigma} \\
q \cdot \mathcal{A}=0 \\
q_{\nu} \mathcal{V}_{\mu}-q_{\mu} \mathcal{V}_{\nu}=\frac{\hbar}{2} \epsilon_{\mu \nu \rho \sigma} \Delta^{\rho} \mathcal{A}^{\sigma}, \\
\left(q^{2}-m^{2}\right) \mathcal{A}^{\mu}=\frac{\hbar}{2} \epsilon^{\mu \nu \rho \sigma} q_{\sigma} \Delta_{\nu} \mathcal{V}_{\rho}, \\
q \cdot \Delta \mathcal{A}^{\mu}+F^{\nu \mu} \mathcal{A}_{\nu}=\frac{\hbar}{2} \epsilon^{\mu \nu \rho \sigma} \Delta_{\sigma} \Delta_{\nu} \mathcal{V}_{\rho} \\
=\frac{\hbar}{2} \epsilon^{\mu \nu \rho \sigma}\left(\partial_{\sigma} F_{\beta \nu}\right) \partial_{q}^{\beta} \mathcal{V}_{\rho}, \\
q \cdot \Delta \mathcal{V}^{\mu}+F^{\nu \mu} \mathcal{V}_{\nu}=\frac{\hbar}{2} \epsilon_{\mu \nu \rho \sigma}\left(\partial^{\nu} F^{\beta \rho}\right) \partial_{q \beta} \mathcal{A}^{\sigma},
\end{gathered}
$$

where $\Delta_{\mu}=\partial_{\mu}+F_{\nu \mu} \partial_{q}^{\nu}$. To obtain the right-hand side of Eq. (A33), we employ the following equation,

$$
\begin{aligned}
2 \epsilon_{\nu \mu \rho \sigma}\left(\Pi^{\nu} \Pi^{\rho}\right) \mathcal{A}^{\sigma} & =\frac{1}{6} \epsilon_{\nu \mu \rho \sigma}\left(\partial^{\rho} F^{\beta \nu}+\partial^{\beta} F^{\rho \nu}\right) \partial_{q \beta} \mathcal{A}^{\sigma} \\
& =\frac{1}{2} \epsilon_{\nu \mu \rho \sigma}\left(\partial^{\rho} F^{\beta \nu}\right) \partial_{q \beta} \mathcal{A}^{\sigma}
\end{aligned}
$$

where we derive the second equality above from the relation

$$
\epsilon_{\nu \mu \rho \sigma} \partial^{\beta} F^{\rho \nu}=2 \epsilon_{\nu \mu \rho \sigma} \partial^{\rho} F^{\beta \nu},
$$

led by

$$
\begin{aligned}
\epsilon_{\nu \mu \rho \sigma} \partial^{\beta} F^{\rho \nu} & =\epsilon_{\nu \mu \rho \sigma} \partial^{\beta}\left(\partial^{\rho} A^{\nu}-\partial^{\nu} A^{\rho}\right) \\
& =\epsilon_{\nu \mu \rho \sigma}\left(\partial^{\rho}\left(F^{\beta \nu}+\partial^{\nu} A^{\beta}\right)-\partial^{\beta} \partial^{\nu} A^{\rho}\right) \\
& =\epsilon_{\nu \mu \rho \sigma}\left(\partial^{\rho} F^{\beta \nu}+\frac{1}{2} \partial^{\beta} F^{\rho \nu}\right) .
\end{aligned}
$$

Note that Eq. (A33) is in fact redundant, which can be derived from Eq. (A30). On the other hand, one can further rewrite Eq. (A28) by using Eq. (A30) in a manner similar to Eq. (A31). Accordingly, we shall deal only with the following six master equations,

$$
\begin{gathered}
\Delta \cdot \mathcal{V}=0, \\
\left(q^{2}-m^{2}\right) \mathcal{V}_{\mu}=-\hbar \tilde{F}_{\mu \nu} \mathcal{A}^{\nu} \\
q_{\nu} \mathcal{V}_{\mu}-q_{\mu} \mathcal{V}_{\nu}=\frac{\hbar}{2} \epsilon_{\mu \nu \rho \sigma} \Delta^{\rho} \mathcal{A}^{\sigma}, \\
q \cdot \mathcal{A}=0, \\
\left(q^{2}-m^{2}\right) \mathcal{A}^{\mu}=\frac{\hbar}{2} \epsilon^{\mu \nu \rho \sigma} q_{\sigma} \Delta_{\nu} \mathcal{V}_{\rho}, \\
q \cdot \Delta \mathcal{A}^{\mu}+F^{\nu \mu} \mathcal{A}_{\nu}=\frac{\hbar}{2} \epsilon^{\mu \nu \rho \sigma}\left(\partial_{\sigma} F_{\beta \nu}\right) \partial_{q}^{\beta} \mathcal{V}_{\rho} \\
=\frac{\hbar}{2}\left(\partial_{\alpha} \tilde{F}^{\mu \nu}\right) \partial_{q}^{\alpha} \mathcal{V}_{\nu},
\end{gathered}
$$

where $\tilde{F}^{\mu \nu}=\epsilon^{\mu \nu \alpha \beta} F_{\alpha \beta} / 2$ and where we employ the Schouten identity,

$\eta_{\mu}^{\lambda} \epsilon_{\rho \nu \alpha \beta}-\eta_{\rho}^{\lambda} \epsilon_{\mu \nu \alpha \beta}-\eta_{\nu}^{\lambda} \epsilon_{\rho \mu \alpha \beta}-\eta_{\alpha}^{\lambda} \epsilon_{\rho \nu \mu \beta}-\eta_{\beta}^{\lambda} \epsilon_{\rho \nu \alpha \mu}=0$,

to derive the last equality in Eq. (A42).

\section{APPENDIX B: PERTURBATIVE SOLUTION FOR WIGNER FUNCTIONS}

We will then seek for the perturbative solution for $\mathcal{V}^{\mu}$ and $\mathcal{A}^{\mu}$ from the equations above, for which we take $(\mathcal{V} / \mathcal{A})_{\mu}=$ $(\mathcal{V} / \mathcal{A})_{0 \mu}+\hbar(\mathcal{V} / \mathcal{A})_{1 \mu}+\mathcal{O}\left(\hbar^{2}\right)$. At leading order up to $\mathcal{O}(1)$, from Eqs. (A38) and (A39), it is found that 


$$
\mathcal{V}_{0 \mu}=2 \pi q_{\mu} \delta\left(q^{2}-m^{2}\right) f_{V},
$$

which follows the leading-order kinetic theory led by Eq. (A37),

$$
\delta\left(q^{2}-m^{2}\right) q \cdot \Delta f_{V}=\mathcal{O}(\hbar)
$$

The $2 \pi$ factor in Eq. (B2) is introduced for convention. For the axial part, Eqs. (A40) and (A41) yield

$$
\mathcal{A}_{0}^{\mu}=2 \pi a^{\mu} \delta\left(q^{2}-m^{2}\right) f_{A},
$$

where

$$
a \cdot q=q^{2}-m^{2}
$$

satisfies $q \cdot \mathcal{A}=0$ with the on-shell condition. Now, plugging Eq. (B4) into Eq. (A42), we find

$$
\delta\left(q^{2}-m^{2}\right)\left(q \cdot \Delta\left(a^{\mu} f_{A}\right)+F^{\nu \mu} a_{\nu} f_{A}\right)=\mathcal{O}(\hbar),
$$

which corresponds to the Bargmann-Michel-Telegdi (BMT) equation.

Subsequently, according to Eqs. (A37)-(A42), for the next-to-leading-order solution up to $\mathcal{O}(\hbar)$, we then have to solve

$$
\Delta \cdot \mathcal{V}=0
$$

$$
\left(q^{2}-m^{2}\right) \mathcal{V}_{1 \mu}=-\tilde{F}_{\mu \nu} \mathcal{A}_{0}^{\nu},
$$

$$
q_{\nu} \mathcal{V}_{1 \mu}-q_{\mu} \mathcal{V}_{1 \nu}=\frac{1}{2} \epsilon_{\mu \nu \rho \sigma} \Delta^{\rho} \mathcal{A}_{0}^{\sigma}
$$

$$
q \cdot \mathcal{A}=0
$$

$$
\left(q^{2}-m^{2}\right) \mathcal{A}_{1}^{\mu}=\frac{1}{2} \epsilon^{\mu \nu \rho \sigma} q_{\sigma} \Delta_{\nu} \mathcal{V}_{0 \rho},
$$

$$
\begin{aligned}
q \cdot \Delta \mathcal{A}_{1}^{\mu}+F^{\nu \mu} \mathcal{A}_{1 \nu} & =\frac{1}{2} \epsilon^{\mu \nu \rho \sigma}\left(\partial_{\sigma} F_{\beta \nu}\right) \partial_{q}^{\beta} \mathcal{V}_{0 \rho} \\
& =\frac{1}{2}\left(\partial_{\alpha} \tilde{F}^{\mu \nu}\right) \partial_{q}^{\alpha} \mathcal{V}_{0 \nu} .
\end{aligned}
$$

The vector part $\mathcal{V}_{1}$ can be solved from Eqs. (B7) and (B8) in analogy to the massless case. Here, Eq. (B8) follows the same structure as the massless master equation to solve for the side-jump term. It is found that

$$
\begin{aligned}
\mathcal{V}_{\mu}= & 2 \pi \delta\left(q^{2}-m^{2}\right) q_{\mu} f_{V}+2 \pi \hbar \tilde{F}_{\mu \nu} a^{\nu} \delta^{\prime}\left(q^{2}-m^{2}\right) f_{A} \\
& +2 \pi \hbar \delta\left(q^{2}-m^{2}\right) G_{\mu}
\end{aligned}
$$

where

$$
\begin{aligned}
\delta\left(q^{2}-m^{2}\right) G_{\mu} & =\frac{\delta\left(q^{2}-m^{2}\right)}{2 n \cdot q} \epsilon_{\mu \nu \rho \sigma} n^{\nu} \Delta^{\rho}\left(a^{\sigma} f_{A}\right)-\frac{\delta^{\prime}\left(q^{2}-m^{2}\right)}{n \cdot q} \tilde{F}_{\mu \nu} n^{\nu} q_{\sigma}\left(a^{\sigma} f_{A}\right) \\
& =\frac{\delta\left(q^{2}-m^{2}\right)}{2 n \cdot q}\left(\epsilon_{\mu \nu \rho \sigma} n^{\nu}\left(\Delta^{\rho} a^{\sigma} f_{A}\right)+2 \tilde{F}_{\mu \nu} n^{\nu} f_{A}\right) .
\end{aligned}
$$

Here, $n^{\mu}$ corresponds to a frame vector analogous to the massless case. We employed the relation $\left(q^{2}-m^{2}\right) \delta^{\prime}\left(q^{2}-\right.$ $\left.m^{2}\right)=-\delta\left(q^{2}-m^{2}\right)$ to obtain the last line of Eq. (B13). We will later utilize the solution in Eq. (B12) to derive the scalar kinetic theory from Eq. (B6). Since $G_{\mu}$ reduces to the side-jump term when $m=0$ and contributes to the magnetization currents, we will call $G_{\mu}$ the MC term.

For the axial part $\mathcal{A}_{1 \mu}$, from Eqs. (B9) and (B10), it is found that

$$
\begin{aligned}
\mathcal{A}_{\mu}= & 2 \pi \delta\left(q^{2}-m^{2}\right) a_{\mu} f_{A}+\hbar \tilde{F}_{\mu \nu} q^{\nu} 2 \pi \delta^{\prime}\left(q^{2}-m^{2}\right) f_{V} \\
& +2 \pi \hbar \delta\left(q^{2}-m^{2}\right) H_{\mu},
\end{aligned}
$$

where $q \cdot H=0$. Based on the side-jump term in the massless limit, it is expected that the MC term here will read

$$
H^{\mu}=g \epsilon^{\mu \nu \alpha \beta} q^{\alpha} n^{\beta} \Delta_{\nu} f_{V},
$$

where $g \rightarrow 1 /(2 q \cdot n)$ when $m \rightarrow 0$. However, given that we are unable to fix $g$ by Eqs. (B9) and (B10), we shall implement an alternative way to derive $g$ from the free WFs in the absence of background fields obtained from the free Dirac fields in the following section. According to Eq. (C31), we find that $2 g=\operatorname{sgn}(q \cdot n) /(|q \cdot n|+m)$, assuming that it remains unchanged in the presence of background fields.

\section{APPENDIX C: WIGNER FUNCTIONS FROM DIRAC WAVE FUNCTIONS}

In this section, we employ an alternative method to derive WFs without background fields up to $\mathcal{O}(\hbar)$. In particular, we will utilize the result to determine the MC term in $\mathcal{A}_{\mu}$. We will start with the second quantization of the free Dirac fields [55], 


$$
\begin{aligned}
& \psi(x)=\int \frac{d^{3} p}{(2 \pi)^{3}} \frac{1}{\sqrt{2 E_{\mathbf{p}}}} \sum_{s}\left(u^{s}(p) e^{-i p \cdot x} a_{\mathbf{p}}^{s}+v^{s}(p) e^{i p \cdot x} b_{\mathbf{p}}^{s \dagger}\right), \\
& \bar{\psi}(x)=\int \frac{d^{3} p}{(2 \pi)^{3}} \frac{1}{\sqrt{2 E_{\mathbf{p}}}} \sum_{s}\left(\bar{u}^{s}(p) e^{i p \cdot x} a_{\mathbf{p}}^{s \dagger}+\bar{v}^{s}(p) e^{-i p \cdot x} b_{\mathbf{p}}^{s}\right),
\end{aligned}
$$

where

$$
u^{s}(p)=\left(\begin{array}{c}
\sqrt{p \cdot \sigma} \xi^{s} \\
\sqrt{p \cdot \bar{\sigma}} \xi^{s}
\end{array}\right), \quad v^{s}(p)=\left(\begin{array}{c}
\sqrt{p \cdot \sigma} \eta^{s} \\
-\sqrt{p \cdot \bar{\sigma}} \eta^{s}
\end{array}\right) .
$$

Here, $\sigma^{\mu}$ and $\bar{\sigma}^{\mu}$ are four-dimensional Pauli matrices which satisfy $\sigma^{\mu} \bar{\sigma}^{\nu}+\sigma^{\nu} \bar{\sigma}^{\mu}=\bar{\sigma}^{\mu} \sigma^{\nu}+\bar{\sigma}^{\nu} \sigma^{\mu}=2 \eta^{\mu \nu}$, with the Minkowski matrix $\eta^{\mu \nu}$. For simplicity, we will drop the antifermions,

$$
S^{<}(x, y)=\langle\bar{\psi}(y) \psi(x)\rangle=\int \frac{d^{3} p}{(2 \pi)^{3} \sqrt{2 E_{\mathbf{p}}}} \int \frac{d^{3} p^{\prime}}{(2 \pi)^{3} \sqrt{2 E_{\mathbf{p}^{\prime}}}} \sum_{s, s^{\prime}}\left(u^{s}(p) \bar{u}^{s^{\prime}}\left(p^{\prime}\right)\left\langle a_{\mathbf{p}^{\prime}}^{s^{\prime} \dagger} a_{\mathbf{p}}^{s}\right\rangle e^{i\left(p^{\prime}-p\right) \cdot X-\frac{i}{2}\left(p^{\prime}+p\right) \cdot Y}\right) .
$$

Here, we make a change of coordinates by taking $X=(x+y) / 2$ and $Y=x-y$ in the second equality. We then carry out the Wigner transformation, which yields

$$
\begin{aligned}
\int d^{4} Y e^{i q \cdot Y} S^{<}(x, y) & =\int \frac{d^{3} p_{-}}{(2 \pi)^{3}} \int \frac{d^{3} p_{+}}{(2 \pi)^{3}}(2 \pi)^{4} e^{-i p_{-} \cdot X} \delta^{4}\left(q-p_{+}\right) \sum_{s, s^{\prime}} u^{s}\left(p_{+}+\frac{p_{-}}{2}\right) \bar{u}^{s^{\prime}}\left(p_{+}-\frac{p_{-}}{2}\right)\left\langle a_{p_{+}-\frac{p_{-}}{2}}^{s^{\prime} \dagger} a_{p_{+}+\frac{p_{-}}{2}}^{s}\right\rangle \\
& =\pi \int \frac{d^{3} p_{-}}{(2 \pi)^{3}} \frac{e^{-i p_{-} \cdot X} \delta\left(q_{0}-p_{+0}\right)}{\left(\left(|\mathbf{q}|^{2}+\frac{\left|\mathbf{p}_{-}\right|^{2}}{4}+m^{2}\right)^{2}-\left(\mathbf{p}_{-} \cdot q\right)^{2}\right)^{1 / 4}} \sum_{s, s^{\prime}} u^{s}\left(p_{+}+\frac{p_{-}}{2}\right) \bar{u}^{s^{\prime}}\left(p_{+}-\frac{p_{-}}{2}\right)\left\langle a_{p_{+}-\frac{p_{-}}{2}}^{s^{\prime}} a_{\boldsymbol{p}_{+}+\frac{p_{-}}{2}}^{s}\right\rangle
\end{aligned}
$$

where $p_{+}=\left(p+p^{\prime}\right) / 2$ and $p_{-}=p-p^{\prime}$. We now define the density operators as

$$
\left\langle a_{\mathbf{p}^{\prime}}^{s^{\prime} \dagger} a_{\mathbf{p}}^{s}\right\rangle=\delta_{s s^{\prime}} N_{V}\left(\mathbf{p}, \mathbf{p}^{\prime}\right)+\mathcal{A}_{s s^{\prime}}\left(\mathbf{p}, \mathbf{p}^{\prime}\right),
$$

where $\mathcal{A}_{s s^{\prime}} \neq 0$ when $s \neq s^{\prime}$, which characterizes certain projection in the spin space. When taking the spin sum, we assign

$$
\begin{aligned}
& \sum_{s} \xi_{s} \xi_{s}^{\dagger}=n \cdot \sigma=n \cdot \bar{\sigma}=I, \\
& \sum_{s, s^{\prime}} \xi_{s} \mathcal{A}_{s s^{\prime}} \xi_{s^{\prime}}^{\dagger}=S\left(\mathbf{p}, \mathbf{p}^{\prime}\right) \cdot \sigma,
\end{aligned}
$$

where $S \cdot n=0$. Consequently, we find

$$
\sum_{s} u^{s}\left(q+\frac{p_{-}}{2}\right) \bar{u}^{s}\left(q-\frac{p_{-}}{2}\right)=\left(\begin{array}{ll}
\sqrt{\sigma \cdot\left(q+\frac{p_{-}}{2}\right) \bar{\sigma} \cdot\left(q-\frac{p_{-}}{2}\right)} & \sqrt{\sigma \cdot\left(q+\frac{p_{-}}{2}\right) \sigma \cdot\left(q-\frac{p_{-}}{2}\right)} \\
\sqrt{\bar{\sigma} \cdot\left(q+\frac{p_{-}}{2}\right) \bar{\sigma} \cdot\left(q-\frac{p_{-}}{2}\right)} & \sqrt{\bar{\sigma} \cdot\left(q+\frac{p_{-}}{2}\right) \sigma \cdot\left(q-\frac{p_{-}}{2}\right)}
\end{array}\right)
$$

and

$$
\sum_{s, s^{\prime}} u^{s}\left(q+\frac{p_{-}}{2}\right) \mathcal{A}_{s s^{\prime}} \bar{u}^{s^{\prime}}\left(q-\frac{p_{-}}{2}\right)=\left(\begin{array}{ll}
\sqrt{\sigma \cdot\left(q+\frac{p_{-}}{2}\right)} \sigma \cdot S \sqrt{\bar{\sigma} \cdot\left(q-\frac{p_{-}}{2}\right)} & \sqrt{\sigma \cdot\left(q+\frac{p_{-}}{2}\right)} \sigma \cdot S \sqrt{\sigma \cdot\left(q-\frac{p_{-}}{2}\right)} \\
\sqrt{\bar{\sigma} \cdot\left(q+\frac{p_{-}}{2}\right)} \sigma \cdot S \sqrt{\bar{\sigma} \cdot\left(q-\frac{p_{-}}{2}\right)} & \sqrt{\bar{\sigma} \cdot\left(q+\frac{p_{-}}{2}\right)} \sigma \cdot S \sqrt{\sigma \cdot\left(q-\frac{\left.p_{-}\right)}{2}\right)}
\end{array}\right) .
$$

To compute the matrix elements above, we will employ the following tricks for Pauli matrices. We may write

$$
q \cdot \sigma=m \exp \left(\hat{q}_{\perp} \cdot \sigma \theta\right), \quad \theta=\tanh ^{-1}\left(\frac{\left|\mathbf{q}_{\perp}\right|}{E_{q}}\right)
$$


where $\hat{q}_{\perp}^{\mu}=q_{\perp}^{\mu} /\left|\mathbf{q}_{\perp}\right|$, which yields

$$
\begin{aligned}
\sqrt{q \cdot \sigma} & =\sqrt{m}\left(\cosh \frac{\theta}{2}+\hat{q}_{\perp} \cdot \sigma \sinh \frac{\theta}{2}\right) \\
& =\sqrt{\frac{1}{2\left(E_{q}+m\right)}}\left(\left(E_{q}+m\right)+q_{\perp} \cdot \sigma\right) .
\end{aligned}
$$

Hereafter, we will use the subscripts $\perp$ to denote the components perpendicular to the frame vector $n^{\mu}$. That is, $V_{\perp}^{\mu} \equiv V^{\mu}-n \cdot V n^{\mu}$ for an arbitrary $V^{\mu}$. We can now write

$$
\begin{aligned}
& \sqrt{\sigma \cdot q}=\sqrt{\frac{1}{2\left(E_{q}+m\right)}}\left(\chi_{q}+q_{\perp} \cdot \sigma\right), \\
& \sqrt{\bar{\sigma} \cdot q}=\sqrt{\frac{1}{2\left(E_{q}+m\right)}}\left(\chi_{q}-q_{\perp} \cdot \sigma\right),
\end{aligned}
$$

where $\chi_{q}=E_{q}+m$. We then have to utilize the following parametrization,

$$
\begin{aligned}
\tilde{v}_{\mu}^{+} \sigma^{\mu} \equiv & \left(\chi_{p}+p_{\perp} \cdot \sigma\right)\left(\chi_{p^{\prime}}+p_{\perp}^{\prime} \cdot \sigma\right) \\
= & \chi_{p} \chi_{p^{\prime}}-p_{\perp} \cdot p_{\perp}^{\prime}+\left(\chi_{p} p_{\perp \mu}^{\prime}+\chi_{p^{\prime}} p_{\perp \mu}\right) \sigma^{\mu} \\
& -i \epsilon^{\mu \nu \alpha \beta} n_{\alpha} \sigma_{\beta} p_{\perp \mu} p_{\perp \nu}^{\prime},
\end{aligned}
$$

which gives

$$
\tilde{v}^{+} \cdot n \approx 2 E_{q}\left(E_{q}+m\right)+\mathcal{O}\left(p_{-}^{2}\right)
$$

and

$\tilde{v}_{\perp \mu}^{+}=2\left(E_{q}+m\right) q_{\perp \mu}-i \epsilon_{\mu \nu \alpha \beta} n^{\alpha} q^{\beta} p_{-}^{\nu}+\mathcal{O}\left(p_{-}^{2}\right)$.

Similarly, one finds that

$$
\begin{aligned}
\tilde{a}_{\mu}^{+} \sigma^{\mu} \equiv & \left(\chi_{p}+p_{\perp} \cdot \sigma\right) S_{\perp} \cdot \sigma\left(\chi_{p^{\prime}}+p_{\perp}^{\prime} \cdot \sigma\right) \\
= & \chi_{p} \chi_{p^{\prime}} S_{\perp} \cdot \sigma-\left(\chi_{p} p_{\perp \mu}^{\prime}+\chi_{p^{\prime}} p_{\perp \mu}\right) S_{\perp}^{\mu} \\
& -i \epsilon^{\mu \nu \alpha \beta} n_{\alpha} \sigma_{\beta} S_{\mu}\left(\chi_{p} p_{\nu}^{\prime}-\chi_{p^{\prime}} p_{\nu}\right)+p_{\perp} \cdot p_{\perp}^{\prime} S_{\perp} \cdot \sigma \\
& -S_{\perp} \cdot p_{\perp}^{\prime} p_{\perp} \cdot \sigma-S_{\perp} \cdot p_{\perp} p_{\perp}^{\prime} \cdot \sigma-i \epsilon^{\mu \nu \alpha \beta} p_{\mu} p_{\nu}^{\prime} n_{\alpha} S_{\beta},
\end{aligned}
$$

which gives

$\tilde{a}^{+} \cdot n \approx-2\left(E_{q}+m\right) q_{\perp} \cdot S_{\perp}+i \epsilon^{\mu \nu \alpha \beta} q_{\mu} p_{-\nu} n_{\alpha} S_{\beta}+\mathcal{O}\left(p_{-}^{2}\right)$

and

$$
\begin{aligned}
\tilde{a}_{\perp \mu}^{+}= & 2\left(m\left(E_{q}+m\right) S_{\perp \mu}-S_{\perp} \cdot q_{\perp} q_{\perp \mu}\right) \\
& -i \epsilon_{\mu \nu \alpha \beta} n^{\alpha} S^{\beta}\left(\frac{q_{\perp} \cdot p_{-}}{E_{q}} q^{\nu}+\left(E_{q}+m\right) p_{-}^{\nu}\right)+\mathcal{O}\left(p_{-}^{2}\right),
\end{aligned}
$$

where we use $p_{-} \cdot \partial_{q} \chi_{q}=-q_{\perp} \cdot p_{-} / E_{q}$. On the other hand, we also introduce

$$
\begin{aligned}
& \tilde{v}_{\mu}^{-} \sigma^{\mu} \equiv\left(\chi_{p}-p_{\perp} \cdot \sigma\right)\left(\chi_{p^{\prime}}-p_{\perp}^{\prime} \cdot \sigma\right), \\
& \tilde{a}_{\mu}^{-} \sigma^{\mu} \equiv\left(\chi_{p}-p_{\perp} \cdot \sigma\right) S_{\perp} \cdot \sigma\left(\chi_{p^{\prime}}-p_{\perp}^{\prime} \cdot \sigma\right) .
\end{aligned}
$$

In the end, up to $\mathcal{O}\left(p_{-}\right)$, we derive

$\tilde{v}^{ \pm} \cdot n=\left(E_{q}+m\right)^{2}$,

$\tilde{a}^{ \pm} \cdot n=\mp 2\left(E_{q}+m\right) q_{\perp} \cdot S_{\perp}+i \epsilon_{\mu \nu \alpha \beta} n^{\alpha} q^{\mu} p_{-}^{\nu} S_{\beta}$,

$$
\tilde{v}_{\perp \mu}^{ \pm}= \pm 2\left(E_{q}+m\right) q_{\perp \mu}-i \epsilon_{\mu \nu \alpha \beta} n^{\alpha} q^{\beta} p_{-}^{\nu},
$$

and

$$
\begin{aligned}
\tilde{a}_{\perp \mu}^{ \pm}= & 2\left(m\left(E_{q}+m\right) S_{\perp \mu}-S_{\perp} \cdot q_{\perp} q_{\perp \mu}\right) \\
& \mp i \epsilon_{\mu \nu \alpha \beta} n^{\alpha} S^{\beta}\left(\frac{q_{\perp} \cdot p_{-}}{E_{q}} q^{\nu}+\left(E_{q}+m\right) p_{-}^{\nu}\right) .
\end{aligned}
$$

Let us focus on the off-diagonal terms in Eqs. (C7) and (C8) associated with $\mathcal{V}_{\mu} \pm \mathcal{A}_{\mu}$. By utilizing Eqs. (C19)(C21), it is found that

$$
\begin{aligned}
\sigma \cdot(\mathcal{V}-\mathcal{A})= & \pi \int \frac{d^{3} p_{-}}{(2 \pi)^{3}} \frac{e^{-i p_{-} \cdot X} \delta\left(q_{0}-E_{q}\right)}{2 E_{q}\left(E_{q}+m\right)} \\
& \times\left(\tilde{v}^{+} \cdot \sigma N_{V}+\tilde{a}^{+} \cdot \sigma\right), \\
\bar{\sigma} \cdot(\mathcal{V}+\mathcal{A})= & \pi \int \frac{d^{3} p_{-}}{(2 \pi)^{3}} \frac{e^{-i p_{-} \cdot X} \delta\left(q_{0}-E_{q}\right)}{2 E_{q}\left(E_{q}+m\right)} \\
& \times\left(\tilde{v}^{-} \cdot \sigma N_{V}+\tilde{a}^{-} \cdot \sigma\right) .
\end{aligned}
$$

Recall that $N_{V}=N_{V}\left(\boldsymbol{q}+\frac{\boldsymbol{p}_{-}}{2}, \boldsymbol{q}-\frac{\boldsymbol{p}_{-}}{2}\right)$ and $S_{\mu}=S_{\mu}\left(\boldsymbol{q}+\frac{\boldsymbol{p}_{-}}{2}\right.$, $\left.\boldsymbol{q}-\frac{\left.\boldsymbol{p}_{-}\right)}{2}\right)$ in the integrands. Thus, one obtains

$$
\begin{aligned}
n \cdot \mathcal{A}= & \frac{\pi}{2} \int \frac{d^{3} p_{-}}{(2 \pi)^{3}} \frac{e^{-i p_{-} \cdot X} \delta\left(q_{0}-E_{q}\right)}{2 E_{q}\left(E_{q}+m\right)} \\
& \times n^{\mu}\left(\left(\tilde{v}_{\mu}^{-}-\tilde{v}_{\mu}^{+}\right) N_{V}+\left(\tilde{a}_{\mu}^{-}-\tilde{a}_{\mu}^{+}\right)\right) \\
= & 2 \pi \delta\left(q^{2}-m^{2}\right) q_{\perp} \cdot \hat{S}_{\perp},
\end{aligned}
$$

$$
\begin{aligned}
\mathcal{A}_{\perp \mu}= & -\frac{\pi}{2} \int \frac{d^{3} p_{-}}{(2 \pi)^{3}} \frac{e^{-i p_{-} \cdot X} \delta\left(q_{0}-E_{q}\right)}{2 E_{q}\left(E_{q}+m\right)} \\
& \times\left(\left(\tilde{v}_{\perp \mu}^{-}+\tilde{v}_{\perp \mu}^{+}\right) N_{V}+\left(\tilde{a}_{\perp \mu}^{-}+\tilde{a}_{\perp \mu}^{+}\right)\right) \\
= & 2 \pi \delta\left(q^{2}-m^{2}\right)\left[\left(\frac{\hat{S}_{\perp} \cdot q_{\perp}}{E_{q}+m} q_{\perp \mu}-m \hat{S}_{\perp \mu}\right)\right. \\
- & \left.\epsilon_{\mu \nu \alpha \beta} \frac{n^{\alpha} q^{\beta}}{2\left(E_{q}+m\right)} \partial^{\nu} \tilde{f}_{V}\right],
\end{aligned}
$$




$$
\begin{aligned}
n \cdot \mathcal{V}= & \frac{\pi}{2} \int \frac{d^{3} p_{-}}{(2 \pi)^{3}} \frac{e^{-i p_{-} \cdot X} \delta\left(q_{0}-E_{q}\right)}{2 E_{q}\left(E_{q}+m\right)} \\
& \times n^{\mu}\left(\left(\tilde{v}_{\mu}^{-}+\tilde{v}_{\mu}^{+}\right) N_{V}+\left(\tilde{a}_{\mu}^{-}+\tilde{a}_{\mu}^{+}\right)\right) \\
= & 2 \pi \delta\left(q^{2}-m^{2}\right)\left[E_{q} \tilde{f}_{V}+\frac{\epsilon^{\rho \nu \alpha \beta}}{2\left(E_{q}+m\right)} n_{\alpha} q_{\beta} \partial_{\nu} \hat{S}_{\rho}\right] \\
= & 2 \pi \delta\left(q^{2}-m^{2}\right) E_{q} f_{V},
\end{aligned}
$$

and

$$
\begin{aligned}
\mathcal{V}_{\perp \mu}= & -\frac{\pi}{2} \int \frac{d^{3} p_{-}}{(2 \pi)^{3}} \frac{e^{-i p_{-} \cdot X} \delta\left(q_{0}-E_{q}\right)}{2 E_{q}\left(E_{q}+m\right)} \\
& \times\left(\left(\tilde{v}_{\perp \mu}^{-}-\tilde{v}_{\perp \mu}^{+}\right) N_{V}+\left(\tilde{a}_{\perp \mu}^{-}-\tilde{a}_{\perp \mu}^{+}\right)\right) \\
= & 2 \pi \delta\left(q^{2}-m^{2}\right)\left[q_{\perp \mu} \tilde{f}_{V}+\frac{\epsilon_{\mu \nu \alpha \beta} n^{\alpha}}{2\left(E_{q}+m\right)}\right. \\
& \left.\times\left(q^{\nu} \frac{q_{\perp} \cdot \partial}{E_{q}}+\left(E_{q}+m\right) \partial^{\nu}\right) \hat{S}^{\beta}\right] \\
= & 2 \pi \delta\left(q^{2}-m^{2}\right)\left[q_{\perp \mu} f_{V}(q, X)\right. \\
& \left.-\frac{\epsilon_{\mu \nu \alpha \beta} n^{\alpha}}{2 E_{q}} \partial^{\nu}\left(\frac{q \cdot \hat{S}_{q}^{\beta}}{\left(E_{q}+m\right)}-m \hat{S}^{\beta}\right)\right],
\end{aligned}
$$

where

$$
\begin{gathered}
\tilde{f}_{V}(q, X) \equiv \int \frac{d^{3} p_{-}}{(2 \pi)^{3}} N_{V}\left(\boldsymbol{q}+\frac{\boldsymbol{p}_{-}}{2}, \boldsymbol{q}-\frac{\boldsymbol{p}_{-}}{2}\right) e^{-i p_{-} \cdot X}, \\
\hat{S}_{\mu}(q, X) \equiv \int \frac{d^{3} p_{-}}{(2 \pi)^{3}} S_{\mu}\left(\boldsymbol{q}+\frac{\boldsymbol{p}_{-}}{2}, \boldsymbol{q}-\frac{\boldsymbol{p}_{-}}{2}\right) e^{-i p_{-} \cdot X},
\end{gathered}
$$

with

$$
f_{V}(q, X)=\tilde{f}_{V}(q, X)+\frac{\epsilon^{\rho \nu \alpha \beta}}{2 E_{q}\left(E_{q}+m\right)} n_{\alpha} q_{\beta} \partial_{\nu} \hat{S}_{\rho}(q, X) .
$$

Recall that $\hat{S}_{\mu}=\hat{S}_{\perp \mu}$. In the computations above, we have employed Schouten identity (A43).
Finally, by taking

$$
\hat{S} \cdot q_{\perp}=a \cdot n f_{A}, \quad \frac{q \cdot \hat{S}}{E_{q}+m} q_{\perp \mu}-m \hat{S}_{\mu}=a_{\perp \mu} f_{A}
$$

and retrieving the $\hbar$ parameters, we obtain

$$
\begin{aligned}
& \mathcal{A}_{\mu}=2 \pi \delta\left(q^{2}-m^{2}\right)\left(a_{\mu} f_{A}+\hbar \frac{\epsilon_{\mu \nu \alpha \beta} q^{\alpha} n^{\beta}}{2(q \cdot n+m)} \partial^{\nu} f_{V}\right), \\
& \mathcal{V}_{\mu}=2 \pi \delta\left(q^{2}-m^{2}\right)\left(q_{\mu} f_{V}+\hbar \frac{\epsilon_{\mu \nu \alpha \beta} n^{\beta}}{2(q \cdot n)} \partial^{\nu}\left(a^{\alpha} f_{A}\right)\right),
\end{aligned}
$$

where we replace $E_{q}$ with $q \cdot n$. Note that here $q \cdot a=$ $q^{2}-m^{2}$ is indeed satisfied by taking $a \cdot n=q \cdot n+m$. One can now also decompose the spin four vector into $a^{\mu} f_{A}=\left(q^{\mu}+m n^{\mu}\right) f_{A}-\hat{S}^{\mu}$.

In the presence of arbitrary background fields, the analytic solution for Dirac wave functions is unknown. Consequently, we generalize the free solution for axial WFs based on the solution in Eq. (C31) and its connection to the massless result for Weyl fermions. We hence conclude that

$$
\begin{aligned}
\mathcal{A}_{\mu}= & 2 \pi \delta\left(q^{2}-m^{2}\right)\left(a_{\mu} f_{A}+\hbar S_{m(n)}^{\mu \nu} \Delta^{\nu} f_{V}\right) \\
& +\hbar \tilde{F}_{\mu \nu} q^{\nu} 2 \pi \delta^{\prime}\left(q^{2}-m^{2}\right) f_{V}
\end{aligned}
$$

by replacing the $\partial_{\nu}$ operator with the $\Delta^{\nu}$ operator in the magnetization-current term, where

$$
S_{m(n)}^{\mu \nu}=\frac{\epsilon^{\mu \nu \alpha \beta} q_{\alpha} n_{\beta}}{2(q \cdot n+m)} .
$$

\section{APPENDIX D: SCALAR/AXIAL-VECTOR KINETIC EQUATIONS}

Given the perturbative solution for $\mathcal{V}^{\mu}$ up to $\mathcal{O}(\hbar)$ in Eq. (B12), we first derive the SKE from $\Delta \cdot \mathcal{V}=0$ in Eq. (A37). In the derivation, we assume that the frame vector $n_{\mu}$ is independent of the momentum $q$. By performing straightforward computations, we find that

$$
\begin{aligned}
\Delta \cdot \mathcal{V}_{1}= & \frac{2 \pi \delta\left(q^{2}-m^{2}\right) \epsilon^{\mu \nu \rho \sigma}}{2 n \cdot q}\left(\left(\frac{E_{\mu} n_{\nu} a_{\sigma}}{q \cdot n}+(q \cdot n) \partial_{\mu}\left(\frac{n_{\nu} a_{\sigma}}{q \cdot n}\right)-n_{\nu}\left(\partial_{\mu} a_{\sigma}\right)\right) \Delta_{\rho}+n_{\nu} a_{\sigma}\left(\partial_{\mu} F_{\beta \rho}\right) \partial_{q}^{\beta}\right) f_{A} \\
& +\frac{2 \pi \delta\left(q^{2}-m^{2}\right) \epsilon^{\mu \nu \rho \sigma} f_{A}}{2 n \cdot q}\left(\left(\left(\partial_{\mu} n_{\nu}\right)+\frac{E_{\mu}-\left(\partial_{\mu} q \cdot n\right)}{q \cdot n} n_{\nu}\right) \Delta_{\rho}+n_{\nu}\left(\partial_{\mu} F_{\beta \rho}\right) \partial_{q}^{\beta}\right) a_{\sigma} \\
& -\frac{2 \pi \delta^{\prime}\left(q^{2}-m^{2}\right)}{n \cdot q} B^{\mu}\left(q \cdot \Delta \tilde{a}_{\mu}+F_{\nu \mu} \tilde{a}^{\nu}\right)+\frac{2 \pi \tilde{F}^{\mu \nu} \delta\left(q^{2}-m^{2}\right) f_{A}}{q \cdot n}\left(\left(\partial_{\mu} n_{\nu}\right)+\frac{E_{\mu}-\left(\partial_{\mu} q \cdot n\right)}{q \cdot n} n_{\nu}\right)
\end{aligned}
$$




$$
\begin{aligned}
= & 2 \pi \delta\left(q^{2}-m^{2}\right)\left(\frac{E_{\mu} S_{a(n)}^{\mu \nu}}{q \cdot n} \Delta_{\nu}+S_{a(n)}^{\mu \nu}\left(\partial_{\mu} F_{\rho \nu}\right) \partial_{q}^{\rho}+\left(\partial_{\mu} S_{a(n)}^{\mu \nu}\right) \Delta_{\nu}\right) f_{A}-\frac{2 \pi \delta^{\prime}\left(q^{2}-m^{2}\right)}{n \cdot q} B^{\mu}\left(q \cdot \Delta \tilde{a}_{\mu}+F_{\nu \mu} \tilde{a}^{\nu}\right) \\
& +\pi \delta\left(q^{2}-m^{2}\right) \epsilon^{\mu \nu \alpha \beta}\left(\frac{n_{\beta}}{q \cdot n}\left(\left(\partial_{\nu} a_{\alpha}\right) \Delta_{\mu}+\left(\partial_{\mu} F_{\rho \nu}\right)\left(\partial_{q}^{\rho} a_{\alpha}\right)\right)+\Delta_{\mu}\left(\frac{n^{\beta}}{q \cdot n}\right)\left(\left(\Delta_{\nu} a_{\alpha}\right)+F_{\nu \alpha}\right)\right) f_{A},
\end{aligned}
$$

where the electric/magnetic fields are defined in terms of $n^{\mu}$,

$$
\tilde{F}_{\mu \nu}=\frac{1}{2} \epsilon_{\mu \nu \alpha \beta} F^{\alpha \beta}=\epsilon_{\mu \nu \alpha \beta} E^{\alpha} n^{\beta}+B_{\mu} n_{\nu}-B_{\nu} n_{\mu}, \quad F_{\mu \nu}=-\epsilon_{\mu \nu \alpha \beta} B^{\alpha} n^{\beta}+E_{\mu} n_{\nu}-E_{\nu} n_{\mu} .
$$

From Eq. (D1), we derive the SKE

$$
\begin{aligned}
0= & \delta\left(q^{2}-m^{2}\right)\left[q \cdot \Delta f_{V}+\frac{\hbar}{2}\left(\frac{E_{\mu} S_{a(n)}^{\mu \nu}}{q \cdot n} \Delta_{\nu}+S_{a(n)}^{\mu \nu}\left(\partial_{\mu} F_{\rho \nu}\right) \partial_{q}^{\rho}+\left(\partial_{\mu} S_{a(n)}^{\mu \nu}\right) \Delta_{\nu}\right) f_{A}\right] \\
& +\frac{\hbar \delta\left(q^{2}-m^{2}\right) \epsilon^{\mu \nu \alpha \beta}}{4}\left(\frac{n_{\beta}}{q \cdot n}\left(\left(\partial_{\nu} a_{\alpha}\right) \Delta_{\mu}+\left(\partial_{\mu} F_{\rho \nu}\right)\left(\partial_{q}^{\rho} a_{\alpha}\right)\right)+\Delta_{\mu}\left(\frac{n^{\beta}}{q \cdot n}\right)\left(\left(\Delta_{\nu} a_{\alpha}\right)+F_{\nu \alpha}\right)\right) f_{A} \\
& -\frac{\hbar \delta^{\prime}\left(q^{2}-m^{2}\right)}{2 q \cdot n} B^{\mu}\left(q \cdot \Delta\left(a_{\mu} f_{A}\right)+F_{\nu \mu} a^{\nu} f_{A}\right),
\end{aligned}
$$

where

$$
S_{a(n)}^{\mu \nu}=\frac{\epsilon^{\mu \nu \alpha \beta} a_{\alpha} n_{\beta}}{2 q \cdot n} .
$$

Next, we may derive the AKE from the perturbative solution of $\mathcal{A}_{\mu}$ up to $\mathcal{O}(\hbar)$ in Eqs. (C32) and (A42) in the master equations. The computations will be more complicated than in the case with the SKE but straightforward. Nevertheless, in order to make a direct comparison with the massless CKT, the underlying strategy is to isolate the $\hbar$ terms proportional to $q^{\mu}$ and the other terms explicitly proportional to $m$ since we expect that the AKE should reduce to $q^{\mu}$ multiplied by the CKT in the massless limit, as foreseen from the off-shell BMT equation.

From Eqs. (C32) and (A42), we obtain

$$
\begin{aligned}
0= & \delta\left(q^{2}-m^{2}\right)\left[\left(a^{\mu} q \cdot \Delta f_{A}+f_{A}\left(q \cdot \Delta a^{\mu}+F^{\nu \mu} a_{\nu}\right)\right)+\hbar\left(q \cdot \Delta\left(S_{m(n)}^{\mu \nu} \Delta_{\nu} f_{V}\right)+F^{\nu \mu} S_{m(n) \nu \rho} \Delta^{\rho} f_{V}\right)\right. \\
& \left.-\hbar \epsilon^{\mu \nu \rho \sigma}\left(\partial_{\sigma} F_{\beta \nu}\right) q_{\rho} \partial_{q}^{\beta} f_{V}\right]+\hbar \delta^{\prime}\left(q^{2}-m^{2}\right) \tilde{F}^{\mu \nu} q_{\nu} q \cdot \Delta f_{V} .
\end{aligned}
$$

We then rearrange this equation in light of the aforementioned strategy to obtain the form for comparison with the CKT when $m=0$. We shall first evaluate

$$
\begin{aligned}
\delta\left(q^{2}-m^{2}\right) q \cdot \Delta\left(S_{m(n)}^{\mu \nu} \Delta_{\nu} f_{V}\right)= & \delta\left(q^{2}-m^{2}\right)\left(\left(q \cdot \Delta S_{m(n)}^{\mu \nu}\right) \Delta_{\nu} f_{V}+S_{m(n)}^{\mu \nu} q \cdot \Delta\left(\Delta_{\nu} f_{V}\right)\right) \\
= & {\left[\delta\left(q^{2}-m^{2}\right)\left(\left(q \cdot \Delta S_{m(n)}^{\mu \nu}\right) \Delta_{\nu}-S_{m(n)}^{\mu \nu} F_{\rho \nu} \Delta^{\rho}+S_{m(n)}^{\mu \nu}\left(\left(q \cdot \partial F_{\beta \nu}\right)-q^{\rho}\left(\partial_{\nu} F_{\beta \rho}\right)\right) \partial_{q}^{\beta}\right)\right.} \\
& \left.-2 \delta^{\prime}\left(q^{2}-m^{2}\right) q^{\rho} F_{\rho \nu} S_{m(n)}^{\mu \nu} q \cdot \Delta\right] f_{V},
\end{aligned}
$$

where we take

$$
\begin{aligned}
\delta\left(q^{2}-m^{2}\right) q \cdot \Delta\left(\Delta_{\nu} f_{V}\right)= & \delta\left(q^{2}-m^{2}\right)\left(\left(q \cdot \Delta \Delta_{\nu}\right) f_{V}+\Delta_{\nu}\left(q \cdot \Delta f_{V}\right)-\left(\Delta_{\nu} q \cdot \Delta\right) f_{V}\right) \\
= & \delta\left(q^{2}-m^{2}\right)\left(\left(q \cdot \Delta \Delta_{\nu}\right) f_{V}-\left(\Delta_{\nu} q \cdot \Delta\right) f_{V}\right)+\Delta_{\nu}\left(\delta\left(q^{2}-m^{2}\right) q \cdot \Delta f_{V}\right) \\
& -\left(\Delta_{\nu} \delta\left(q^{2}-m^{2}\right)\right)\left(q \cdot \Delta f_{V}\right) .
\end{aligned}
$$

For the first component of Eq. (D6), we find that 


$$
\begin{aligned}
\delta\left(q^{2}-m^{2}\right)\left(q \cdot \Delta S_{m(n)}^{\mu \nu}\right) \Delta_{\nu} f_{V}= & \delta\left(q^{2}-m^{2}\right)\left[q^{\mu}\left(\Delta_{\alpha} S_{m(n)}^{\alpha \nu}\right)+\left(\Delta_{\alpha} S_{m(n)}^{\mu \alpha}\right) q^{\nu}+\epsilon^{\mu \nu \alpha \sigma}\left(\frac{q^{\rho} F_{\rho \alpha} n_{\sigma}}{2(q \cdot n+m)}+q^{2}\left(\partial_{\alpha} \frac{n_{\sigma}}{2(q \cdot n+m)}\right)\right)\right. \\
& \left.+\frac{\epsilon^{\mu \nu \alpha \sigma} q^{2} n_{\sigma} E_{\alpha}}{2(q \cdot n+m)^{2}}+\epsilon^{\mu \nu \rho \alpha}\left(\frac{q \cdot n F_{\rho \alpha}}{2(q \cdot n+m)}+q_{\rho}\left(\partial_{\alpha} \frac{q \cdot n}{2(q \cdot n+m)}\right)\right)+\frac{\epsilon^{\mu \nu \rho \alpha} q \cdot n q_{\rho} E_{\alpha}}{2(q \cdot n+m)^{2}}\right] \Delta_{\nu} f_{V} \\
= & \delta\left(q^{2}-m^{2}\right)\left[q^{\mu}\left(\partial_{\alpha} S_{m(n)}^{\alpha \nu}\right)+q^{\mu} \frac{S_{m(n)}^{\alpha \nu} E_{\alpha}}{q \cdot n+m}+\frac{\left(q \cdot n \tilde{F}^{\mu \nu}+q^{\mu} B^{\nu}\right)}{2(q \cdot n+m)}+\frac{\epsilon^{\mu \nu \rho \alpha} q_{\rho} E_{\alpha}}{2(q \cdot n+m)}\right. \\
& \left.+\frac{\epsilon^{\mu \nu \alpha \beta} m^{2}}{2(q \cdot n+m)}\left(\partial_{\alpha} n_{\beta}\right)+\frac{\epsilon^{\mu \nu \alpha \beta}\left(m^{2} n_{\beta}+m q_{\beta}\right)}{2(q \cdot n+m)^{2}}\left(E_{\alpha}-\partial_{\alpha}(q \cdot n)\right)\right] \Delta_{\nu} f_{V}+\mathcal{O}(\hbar),
\end{aligned}
$$

where we use

$$
\begin{aligned}
\epsilon^{\mu \nu \alpha \sigma} F_{\rho \alpha} & =\delta_{\rho}^{\mu} B^{[\sigma} n^{\nu]}+\delta_{\rho}^{\sigma} B^{[\nu} n^{\mu]}+\delta_{\rho}^{\nu} B^{[\mu} n^{\sigma]}+\epsilon^{\mu \nu \alpha \sigma} E_{[\rho} n_{\alpha]} \\
& =\delta_{\rho}^{\mu} \tilde{F}^{\sigma \nu}+\delta_{\rho}^{\sigma} \tilde{F}^{\nu \mu}+\delta_{\rho}^{\nu} \tilde{F}^{\mu \sigma}
\end{aligned}
$$

and

$$
\begin{aligned}
\Delta_{\alpha} S_{m(n)}^{\alpha \nu} & =\partial_{\alpha} S_{m(n)}^{\alpha \nu}+\epsilon^{\alpha \nu \rho \sigma} F_{\beta \alpha} \partial_{q}^{\beta}\left(\frac{q_{\rho} n_{\sigma}}{2(q \cdot n+m)}\right) \\
& =\partial_{\alpha} S_{m(n)}^{\alpha \nu}+\frac{B^{\nu}}{q \cdot n+m}+\frac{S_{m(n)}^{\alpha \nu} E_{\alpha}}{q \cdot n+m} .
\end{aligned}
$$

Subsequently, the second component in Eq. (D6) reads

$$
\begin{aligned}
& -\delta\left(q^{2}-m^{2}\right) S_{m(n)}^{\mu \nu} F_{\rho \nu} \Delta^{\rho} f_{V} \\
& =-\frac{\delta\left(q^{2}-m^{2}\right)}{2(q \cdot n+m)} \epsilon^{\mu \nu \alpha \beta} q_{\alpha} n_{\beta} F_{\rho \nu} \Delta^{\rho} f_{V} \\
& =\frac{\delta\left(q^{2}-m^{2}\right)}{2(q \cdot n+m)}\left(\delta_{\rho}^{\mu} \tilde{F}^{\beta \alpha}+\delta_{\rho}^{\alpha} \tilde{F}^{\mu \beta}+\delta_{\rho}^{\beta} \tilde{F}^{\alpha \mu}\right) q_{\alpha} n_{\beta} \Delta^{\rho} f_{V} \\
& =\frac{\delta\left(q^{2}-m^{2}\right)}{2(q \cdot n+m)}\left(-q \cdot B \Delta^{\mu}+q_{\alpha} \tilde{F}^{\alpha \mu} n \cdot \Delta\right) f_{V} .
\end{aligned}
$$

Next, the third component in Eq. (D6) can be written as

$$
\begin{aligned}
& \delta\left(q^{2}-m^{2}\right) S_{m(n)}^{\mu \nu}\left(\left(q \cdot \partial F_{\beta \nu}\right)-q^{\rho}\left(\partial_{\nu} F_{\beta \rho}\right)\right) \partial_{q}^{\beta} f_{V} \\
&= \delta\left(q^{2}-m^{2}\right) S_{m(n)}^{\mu \nu}\left(q \cdot \partial F_{\beta \nu}\right) \partial_{q}^{\beta} f_{V}-\frac{\delta\left(q^{2}-m^{2}\right)}{2(q \cdot n+m)}\left(q^{\mu} \epsilon^{\rho \nu \alpha \sigma}\right. \\
&\left.+q^{\nu} \epsilon^{\mu \rho \alpha \sigma}+q^{\alpha} \epsilon^{\mu \nu \rho \sigma}+q^{\sigma} \epsilon^{\mu \nu \alpha \rho}\right) q_{\alpha} n_{\sigma}\left(\partial_{\nu} F_{\beta \rho}\right) \partial_{q}^{\beta} f_{V} \\
&=-\delta\left(q^{2}-m^{2}\right)\left(q^{\mu} S_{m(n)}^{\rho \nu}\left(\partial_{\nu} F_{\beta \rho}\right)+\frac{m^{2} \epsilon^{\mu \nu \rho \sigma} n_{\sigma}}{2(q \cdot n+m)}\left(\partial_{\nu} F_{\beta \rho}\right)\right. \\
&\left.-\left(1-\frac{m}{q \cdot n+m}\right) \frac{\epsilon^{\mu \nu \rho \sigma} q_{\rho}}{2}\left(\partial_{\sigma} F_{\beta \nu}\right)\right) \partial_{q}^{\beta} f_{V} .
\end{aligned}
$$

For the forth component in Eq. (D6), it is found

$$
\begin{aligned}
- & \delta^{\prime}\left(q^{2}-m^{2}\right) q^{\rho} F_{\rho \nu} S_{m(n)}^{\mu \nu} q \cdot \Delta f_{V} \\
= & -\frac{\delta^{\prime}\left(q^{2}-m^{2}\right)}{2(q \cdot n+m)} q^{\rho} F_{\rho \nu} \epsilon^{\mu \nu \alpha \beta} q_{\alpha} n_{\beta} q \cdot \Delta f_{V} \\
= & -\frac{\delta^{\prime}\left(q^{2}-m^{2}\right)}{2(q \cdot n+m)} F_{\rho \nu}\left(q^{\mu} \epsilon^{\rho \nu \alpha \beta}+q^{\nu} \epsilon^{\mu \rho \alpha \beta}+q^{\alpha} \epsilon^{\mu \nu \rho \beta}\right. \\
& \left.+q^{\beta} \epsilon^{\mu \nu \alpha \rho}\right) q_{\alpha} n_{\beta} q \cdot \Delta f_{V} \\
= & -\left[q^{\mu} q \cdot B \frac{\delta^{\prime}\left(q^{2}-m^{2}\right)}{q \cdot n+m}-\delta^{\prime}\left(q^{2}-m^{2}\right) q^{\rho} F_{\rho \nu} S_{m(n)}^{\mu \nu}\right. \\
& \left.-\frac{\delta^{\prime}\left(q^{2}-m^{2}\right)}{q \cdot n+m} \tilde{F}^{\mu \beta}\left(q^{2} n_{\beta}-q \cdot n q_{\beta}\right)\right] q \cdot \Delta f_{V},
\end{aligned}
$$

which yields

$$
\begin{aligned}
- & \delta^{\prime}\left(q^{2}-m^{2}\right) q^{\rho} F_{\rho \nu} S_{m(n)}^{\mu \nu} \frac{q \cdot \Delta f_{V}}{2} \\
= & -\left[q^{\mu} q \cdot B \frac{\delta^{\prime}\left(q^{2}-m^{2}\right)}{q \cdot n+m}-\frac{\delta^{\prime}\left(q^{2}-m^{2}\right)}{q \cdot n+m}\left(m^{2} B^{\mu}\right.\right. \\
& \left.\left.-q \cdot n q_{\beta} \tilde{F}^{\mu \beta}\right)\right] q \cdot \Delta f_{V}+\mathcal{O}(\hbar) \\
= & -\frac{\delta^{\prime}\left(q^{2}-m^{2}\right)}{2}\left[\frac{q^{\mu} q \cdot B}{q \cdot n+m}-\frac{m\left(m B^{\mu}+q_{\beta} \tilde{F}^{\mu \beta}\right)}{q \cdot n+m}\right. \\
& \left.+q_{\nu} \tilde{F}^{\mu \nu}\right] q \cdot \Delta f_{V}+\mathcal{O}(\hbar) .
\end{aligned}
$$

On the other hand, one finds that

$$
\begin{aligned}
& \delta\left(q^{2}-m^{2}\right) F^{\nu \mu} S_{m(n) \nu \rho} \Delta^{\rho} f_{V} \\
& =\delta\left(q^{2}-m^{2}\right) \frac{\epsilon_{\rho \nu \alpha \sigma} F^{\mu \alpha}}{2(q \cdot n+m)} q^{\sigma} n^{\nu} \Delta^{\rho} f_{V} \\
& =\frac{\delta\left(q^{2}-m^{2}\right)}{2(q \cdot n+m)}\left[q \cdot B \Delta^{\mu}-q^{\mu} B \cdot \Delta+n^{\mu} q^{\rho} \tilde{F}_{\nu \rho} \Delta^{\nu}\right] f_{V} .
\end{aligned}
$$

Combining all pieces, we acquire 


$$
\begin{aligned}
\delta & \left(q^{2}-m^{2}\right)\left[q \cdot \Delta\left(S_{m(n)}^{\mu \nu} \Delta_{\nu} f_{V}\right)+F^{\nu \mu} S_{m(n) \nu \rho} \Delta^{\rho} f_{V}\right]+\delta^{\prime}\left(q^{2}-m^{2}\right) \tilde{F}^{\mu \nu} q_{\nu} q \cdot \Delta f_{V}-\frac{\epsilon^{\mu \nu \rho \sigma}}{2}\left(\partial_{\sigma} F_{\beta \nu}\right) q_{\rho} \partial_{q}^{\beta} f_{V} \\
= & \delta\left(q^{2}-m^{2}\right)\left[q^{\mu}\left(\partial_{\alpha} S_{m(n)}^{\alpha \nu}\right)+q^{\mu} \frac{S_{m(n)}^{\alpha \nu} E_{\alpha}}{q \cdot n+m}+\frac{\left(q \cdot n \tilde{F}^{\mu \nu}+q_{\rho} \tilde{F}^{\rho \mu} n^{\nu}+n^{\mu} q_{\rho} \tilde{F}^{\nu \rho}\right)}{2(q \cdot n+m)}+\frac{\epsilon^{\mu \nu \rho \alpha} q_{\rho} E_{\alpha}}{2(q \cdot n+m)}\right. \\
& \left.+\frac{\epsilon^{\mu \nu \alpha \beta}\left(m^{2} n_{\beta}+m q_{\beta}\right)}{2(q \cdot n+m)^{2}}\left(E_{\alpha}-\partial_{\alpha}(q \cdot n)\right)\right] \Delta_{\nu} f_{V}-\delta^{\prime}\left(q^{2}-m^{2}\right)\left[\frac{q^{\mu} q \cdot B}{q \cdot n+m}-\frac{m\left(m B^{\mu}+q_{\beta} \tilde{F}^{\mu \beta}\right)}{q \cdot n+m}\right] q \cdot \Delta f_{V} \\
& +\delta\left(q^{2}-m^{2}\right)\left[q^{\mu} S_{m(n)}^{\rho \nu}\left(\partial_{\rho} F_{\beta \nu}\right)+\frac{\epsilon^{\mu \nu \rho \sigma} m\left(m n_{\rho}+q_{\rho}\right)}{2(q \cdot n+m)}\left(\partial_{\nu} F_{\beta \sigma}\right)\right] \partial_{q}^{\beta} f_{V} \\
= & q^{\mu}\left\{\delta\left(q^{2}-m^{2}\right)\left[\left(\partial_{\alpha} S_{m(n)}^{\alpha \nu}\right) \Delta_{\nu}+\frac{S_{m(n)}^{\alpha \nu} E_{\alpha} \Delta_{\nu}}{q \cdot n+m}+S_{m(n)}^{\rho \nu}\left(\partial_{\rho} F_{\beta \nu}\right) \partial_{q}^{\beta}\right]-\delta^{\prime}\left(q^{2}-m^{2}\right) \frac{q \cdot B}{q \cdot n+m} q^{\prime} \Delta\right\} f_{V} \\
& +m\left\{\delta\left(q^{2}-m^{2}\right) \frac{\epsilon^{\mu \nu \alpha \beta}\left(m n_{\beta}+q \beta\right.}{2(q \cdot n+m)}\left(\frac{\left(E_{\alpha}-\partial_{\alpha}(q \cdot n)\right)}{q \cdot n+m} \Delta_{\nu}-\left(\partial_{\nu} F_{\rho \alpha}\right) \partial_{q}^{\rho}\right)\right. \\
& \left.+\delta^{\prime}\left(q^{2}-m^{2}\right) \frac{\left(m B^{\mu}+q_{\beta} \tilde{F}^{\mu \beta}\right)}{q \cdot n+m} q \cdot \Delta\right\} f_{V} \cdot
\end{aligned}
$$

To obtain the last equality above, we apply

$$
\begin{aligned}
q \cdot n \tilde{F}^{\mu \nu}+q_{\rho} \tilde{F}^{\rho \mu} n^{\nu}+n^{\mu} q_{\rho} \tilde{F}^{\nu \rho}+\epsilon^{\mu \nu \rho \alpha} q_{\rho} E_{\alpha}= & q \cdot n\left(B^{[\mu} n^{\nu]}+\epsilon^{\mu \nu \alpha \beta} E_{\alpha} n_{\beta}\right)+q \cdot B n^{\mu} n^{\nu}-q \cdot n B^{\mu} n^{\nu} \\
& +\epsilon^{\rho \mu \alpha \beta} q_{\rho} E_{\alpha} n_{\beta} n^{\nu}+n^{\mu}\left(B^{\nu} q \cdot n-q \cdot B n^{\nu}\right)+n^{\mu} \epsilon^{\nu \rho \alpha \beta} q_{\rho} E_{\alpha} n_{\beta}+\epsilon^{\mu \nu \rho \alpha} q_{\rho} E_{\alpha} \\
= & \epsilon^{\mu \nu \alpha \beta}\left(q \cdot n E_{\alpha} n_{\beta}-E_{\alpha} q_{\beta}\right)+q_{\rho} E_{\alpha} n_{\beta}\left(\epsilon^{\rho \mu \alpha \beta} n^{\nu}-\epsilon^{\rho \nu \alpha \beta} n^{\mu}\right) \\
= & 0
\end{aligned}
$$

where we also use

$$
q_{\rho} E_{\alpha} n_{\beta} \epsilon^{\rho \nu \alpha \beta} n^{\mu}=(q \cdot n) E_{\alpha} n_{\beta} \epsilon^{\mu \nu \alpha \beta}+q_{\rho} E_{\alpha} n_{\beta} \epsilon^{\rho \mu \alpha \beta} n^{\nu}+\epsilon^{\rho \nu \alpha \mu} q_{\rho} E_{\alpha} .
$$

From Eq. (D5), the AKE takes the form

$$
\begin{aligned}
0= & \delta\left(q^{2}-m^{2}\right)\left(a^{\mu} q \cdot \Delta f_{A}+f_{A}\left(q \cdot \Delta a^{\mu}+F^{\nu \mu} a_{\nu}\right)\right)+\hbar q^{\mu}\left\{\delta ( q ^ { 2 } - m ^ { 2 } ) \left[\left(\partial_{\alpha} S_{m(n)}^{\alpha \nu}\right) \Delta_{\nu}+\frac{S_{m(n)}^{\alpha \nu} E_{\alpha} \Delta_{\nu}}{q \cdot n+m}\right.\right. \\
& \left.\left.+S_{m(n)}^{\rho \nu}\left(\partial_{\rho} F_{\beta \nu}\right) \partial_{q}^{\beta}\right]-\delta^{\prime}\left(q^{2}-m^{2}\right) \frac{q \cdot B}{q \cdot n+m} q \cdot \Delta\right\} f_{V} \\
& +\hbar m\left\{\delta\left(q^{2}-m^{2}\right) \frac{\epsilon^{\mu \nu \alpha \beta}\left(m n_{\beta}+q_{\beta}\right)}{2(q \cdot n+m)}\left(\frac{\left(E_{\alpha}-\partial_{\alpha}(q \cdot n)\right)}{q \cdot n+m} \Delta_{\nu}-\left(\partial_{\nu} F_{\rho \alpha}\right) \partial_{q}^{\rho}\right)\right. \\
& \left.+\delta^{\prime}\left(q^{2}-m^{2}\right) \frac{\left(m B^{\mu}+q_{\beta} \tilde{F}^{\mu \beta}\right)}{q \cdot n+m} q \cdot \Delta\right\} f_{V} \cdot
\end{aligned}
$$

\section{APPENDIX E: SPIN HALL EFFECT}

We show how Eq. (D19) reveals a spin Hall effect in a nonrelativistic case. Assuming that $E^{\mu}$ and $n^{\mu}$ are constant and approximating $q^{\mu} \approx m n^{\nu}$, Eq. (D19) reduces to

$$
\begin{aligned}
& \delta\left(q^{2}-m^{2}\right)\left(\square^{\mu \nu} \tilde{a}_{\nu}+\frac{\hbar}{4} \epsilon^{\mu \nu \alpha \beta} E_{\alpha} n_{\beta} \Delta_{\nu} f_{V}\right) \\
& +\frac{\hbar}{2} \delta^{\prime}\left(q^{2}-m^{2}\right) \epsilon^{\mu \nu \alpha \beta} E_{\alpha} n_{\beta} q_{\nu} q \cdot \Delta f_{V} \approx 0,
\end{aligned}
$$

where $\square^{\mu \nu}=\eta^{\mu \nu} q \cdot \Delta+F^{\nu \mu}$ and $\tilde{a}^{\nu}=a^{\nu} f_{A}$. By using

$$
\begin{aligned}
& \delta^{\prime}\left(q^{2}-m^{2}\right) \epsilon^{\mu \nu \alpha \beta} E_{\alpha} n_{\beta} q_{\nu} q \cdot \Delta f_{V} \\
& =-\frac{\delta\left(q^{2}-m^{2}\right)}{2} \epsilon^{\mu \nu \alpha \beta} E_{\alpha} n_{\beta}\left(\Delta_{\nu}+q \cdot \Delta \partial_{q \nu}\right) f_{V}+\mathcal{O}(\hbar),
\end{aligned}
$$

Eq. (E1) becomes 
$\delta\left(q^{2}-m^{2}\right)\left[q \cdot \Delta\left(\tilde{a}^{\mu}-\frac{\hbar \epsilon^{\mu \nu \alpha \beta}}{4} E_{\alpha} n_{\beta} \partial_{q \nu} f_{V}\right)+F^{\nu \mu} \tilde{a}_{\nu}\right] \approx 0$,

which can be written as

$$
\begin{aligned}
& n \cdot \Delta\left(\tilde{a}^{\mu}-\frac{\hbar \epsilon^{\mu \nu \alpha \beta}}{4} E_{\alpha} n_{\beta} \partial_{q \nu} f_{V}\right) \\
& =\left(n \cdot \partial+E_{\rho} \partial_{q}^{\rho}\right)\left(\tilde{a}^{\mu}-\frac{\hbar \epsilon^{\mu \nu \alpha \beta}}{4} E_{\alpha} n_{\beta} \partial_{q \nu} f_{V}\right) \approx 0
\end{aligned}
$$

by further dropping the $\mathcal{O}(1 / m)$ suppression terms. On the other hand, in such a limit, the axial WF approximately reads

$$
\begin{aligned}
\mathcal{A}_{\mu} \approx & 2 \pi \delta\left(q^{2}-m^{2}\right) a_{\mu} f_{A} \\
& +\hbar \pi \epsilon_{\mu \nu \alpha \beta} E^{\alpha} n^{\beta}\left(\partial_{q}^{\nu} \delta\left(q^{2}-m^{2}\right)\right) f_{V} .
\end{aligned}
$$

In a stationary state such that

$$
\tilde{a}^{\mu}=\frac{\hbar}{4} \epsilon^{\mu \nu \alpha \beta} E_{\alpha} n_{\beta} \partial_{q \nu} f_{V},
$$

we find that

$$
\begin{aligned}
J_{5}^{\mu} & =4 \int \frac{d^{4} q}{(2 \pi)^{4}} \mathcal{A}^{\mu} \\
& \approx 4 \pi \int \frac{d^{4} q}{(2 \pi)^{4}} \delta\left(q^{2}-m^{2}\right)\left(2 \tilde{a}^{\mu}-\hbar \epsilon^{\mu \nu \alpha \beta} E_{\alpha} n_{\beta} \partial_{q \nu} f_{V}\right) \\
& =-2 \pi \hbar \epsilon^{\mu \nu \alpha \beta} E_{\alpha} n_{\beta} \int \frac{d^{4} q}{(2 \pi)^{4}} \delta\left(q^{2}-m^{2}\right) \partial_{q \nu} f_{V} .
\end{aligned}
$$

\section{APPENDIX F: FRAME INDEPENDENCE}

In this section, we derive the modified frame transformation upon $f_{V}$ and $a^{\mu} f_{A}$ at $\mathcal{O}(\hbar)$ to ensure the frame independence of $\mathcal{V}^{\mu}$ and $\mathcal{A}^{\mu}$. Recall that the explicit form of $\mathcal{V}^{\mu}$ and $\mathcal{A}^{\mu}$ in an arbitrary frame $n^{\mu}$ reads

$$
\begin{gathered}
\mathcal{V}^{\mu}=2 \pi \delta\left(q^{2}-m^{2}\right)\left[q^{\mu} f_{V}^{(n)}+\frac{\hbar \epsilon^{\mu \nu \rho \sigma} n_{\nu}}{2 q \cdot n}\left(\Delta_{\rho}\left(a_{\sigma}^{(n)} f_{A}^{(n)}\right)+F_{\rho \sigma} f_{A}^{(n)}\right)\right]+2 \pi \hbar \tilde{F}^{\mu \nu} a_{\nu}^{(n)} \delta^{\prime}\left(q^{2}-m^{2}\right) f_{A}^{(n)}, \\
\mathcal{A}^{\mu}=2 \pi \delta\left(q^{2}-m^{2}\right)\left[a^{(n) \mu} f_{A}^{(n)}+\frac{\hbar \epsilon^{\mu \nu \alpha \beta} q_{\alpha} n_{\beta}}{2(q \cdot n+m)} \Delta_{\nu} f_{V}^{(n)}\right]+2 \pi \hbar \tilde{F}^{\mu \nu} q_{\nu} \delta^{\prime}\left(q^{2}-m^{2}\right) f_{V}^{(n)},
\end{gathered}
$$

where we further add the superscripts $(n)$ on $f_{V}$ and $a^{\mu} f_{A}$ to highlight their frame dependence due to the presence of magnetization terms. Based on the frame independence of $\mathcal{V}^{\mu}$, we obtain

$$
\delta\left(q^{2}-m^{2}\right)\left[q^{\mu}\left(f_{V}^{(n)}-f_{V}^{\left(n^{\prime}\right)}\right)+\hbar \epsilon^{\mu \nu \rho \sigma}\left(\frac{n_{\nu}}{2 q \cdot n}-\frac{n_{\nu}^{\prime}}{2 q \cdot n^{\prime}}\right)\left(\Delta_{\rho}\left(a_{\sigma} f_{A}\right)+F_{\rho \sigma} f_{A}\right)\right]=0
$$

up to $\mathcal{O}(\hbar)$ when considering the frame transformation from $n^{\mu}$ to $n^{\prime \mu}$, where we drop the frame dependence on $a^{\mu}$ and $f_{A}$ therein since only their frame independent part $\mathcal{O}\left(\hbar^{0}\right)$ contributes. Contracting Eq. (F3) with $n^{\mu}$, one immediately obtains

$$
f_{V}^{\left(n^{\prime}\right)}=f_{V}^{(n)}+\frac{\hbar \epsilon^{\lambda \nu \rho \sigma} n_{\lambda} n_{\nu}^{\prime}}{2(q \cdot n)\left(q \cdot n^{\prime}\right)}\left(\Delta_{\rho}\left(a_{\sigma} f_{A}\right)+F_{\rho \sigma} f_{A}\right)
$$

as the modified frame transformation of $f_{V}$. One may show that Eq. (F4) indeed satisfies Eq. (F3) explicitly. By using Eq. (F4) and Schouten identity (A43), it is found that

$$
\begin{aligned}
& \delta\left(q^{2}-m^{2}\right) q^{\mu}\left(f_{V}^{(n)}-f_{V}^{\left(n^{\prime}\right)}\right) \\
& =\hbar \delta\left(q^{2}-m^{2}\right)\left(\epsilon^{\mu \nu \rho \sigma} q \cdot n n_{\nu}^{\prime}+\epsilon^{\lambda \mu \rho \sigma} n_{\lambda} q \cdot n^{\prime}+\epsilon^{\lambda \nu \mu \sigma} n_{\lambda} n_{\nu}^{\prime} q^{\rho}+\epsilon^{\lambda \nu \rho \mu} n_{\lambda} n_{\nu}^{\prime} q^{\sigma}\right) \frac{\left(\Delta_{\rho}\left(a_{\sigma} f_{A}\right)+F_{\rho \sigma} f_{A}\right)}{2(q \cdot n)\left(q \cdot n^{\prime}\right)} \\
& =\hbar \delta\left(q^{2}-m^{2}\right)\left[\epsilon^{\mu \nu \rho \sigma}\left(\frac{n_{\nu}^{\prime}}{2 q \cdot n^{\prime}}-\frac{n_{\nu}}{2 q \cdot n}\right)\left(\Delta_{\rho}\left(a_{\sigma} f_{A}\right)+F_{\rho \sigma} f_{A}\right)+\frac{\epsilon^{\lambda \nu \mu \sigma}}{2(q \cdot n)\left(q \cdot n^{\prime}\right)}\left(q \cdot \Delta\left(a_{\sigma} f_{A}\right)+F_{\rho \sigma} a^{\sigma} f_{A}\right)\right],
\end{aligned}
$$

where we employ $q^{\rho} \Delta_{\sigma}\left(a_{\rho} f_{A}\right)=\Delta_{\sigma}\left(q \cdot a f_{A}\right)-F_{\rho \sigma} a^{\rho} f_{A}$ and $q \cdot a=q^{2}-m^{2}$ in the computation. Since

$$
\hbar \delta\left(q^{2}-m^{2}\right)\left(q \cdot \Delta\left(a_{\sigma} f_{A}\right)+F_{\rho \sigma} a^{\sigma} f_{A}\right)=\mathcal{O}\left(\hbar^{2}\right)
$$

according to the AKE and the corresponding term thus can be dropped in Eq. (F5), Eq. (F3) is indeed satisfied by the modified frame transformation. For the frame independence of $\mathcal{A}^{\mu}$, it is straightforward to find that 


$$
\begin{aligned}
& a^{\left(n^{\prime}\right) \mu} f_{A}^{\left(n^{\prime}\right)}-a^{(n) \mu} f_{A}^{(n)} \\
& =\hbar \epsilon^{\mu \nu \alpha \beta}\left(\frac{n_{\beta}}{2(q \cdot n+m)}-\frac{n_{\beta}^{\prime}}{2\left(q \cdot n^{\prime}+m\right)}\right) q_{\alpha} \Delta_{\nu} f_{V}
\end{aligned}
$$

as the modified frame transformation. From Eq. (F4), one can make the connection between $n^{\mu}=n^{\mu}(X)$ and the rest frame $n_{r}^{\mu}=q^{\mu} / m$ through

$$
f_{V}^{\left(n_{r}\right)}=f_{V}^{(n)}+\frac{\hbar \epsilon^{\lambda \nu \rho \sigma} n_{\lambda} q_{\nu}}{2(q \cdot n) m^{2}}\left(\Delta_{\rho}\left(a_{\sigma} f_{A}\right)+F_{\rho \sigma} f_{A}\right) .
$$

Nonetheless, in the small-mass region, $f_{V}^{\left(n_{r}\right)}$ contains a divergent term. For $\mathcal{V}^{\mu}$ to be frame invariant, such a divergent term from the modified frame transformation should cancel the divergent part of the magnetization current in $n_{r}^{\mu}$ so that the remaining finite part agrees with the magnetization current obtained in $n^{\mu}(X)$. One is forced to deal with such a subtle cancellation caused by an inappropriate frame choice when $m$ is smaller than the gradient or electromagnetic scales. Thanks to our results in the general frame, we may discuss the frame transformation property and find how the frame invariance should be realized. Even better, we can choose an appropriate frame to avoid such pathological behavior. However, without knowing such a general frame transformation, naively working in the rest frame cannot correctly capture the finite quantum effect when $m$ is small.
[1] A. Vilenkin, Phys. Rev. D 20, 1807 (1979).

[2] D. E. Kharzeev, L. D. McLerran, and H. J. Warringa, Nucl. Phys. A803, 227 (2008).

[3] K. Fukushima, D. E. Kharzeev, and H. J. Warringa, Phys. Rev. D 78, 074033 (2008).

[4] D. E. Kharzeev, J. Liao, S. A. Voloshin, and G. Wang, Prog. Part. Nucl. Phys. 88, 1 (2016).

[5] K. Hattori and X.-G. Huang, Nucl. Sci. Technol. 28, 26 (2017).

[6] Q. Li, D. E. Kharzeev, C. Zhang, Y. Huang, I. Pletikosic, A. V. Fedorov, R. D. Zhong, J. A. Schneeloch, G. D. Gu, and T. Valla, Nat. Phys. 12, 550 (2016).

[7] N. Yamamoto, Phys. Rev. D 93, 065017 (2016).

[8] Y. Masada, K. Kotake, T. Takiwaki, and N. Yamamoto, Phys. Rev. D 98, 083018 (2018).

[9] J.-H. Gao, Z.-T. Liang, S. Pu, Q. Wang, and X.-N. Wang, Phys. Rev. Lett. 109, 232301 (2012).

[10] D. T. Son and N. Yamamoto, Phys. Rev. Lett. 109, 181602 (2012).

[11] M. Stephanov and Y. Yin, Phys. Rev. Lett. 109, 162001 (2012).

[12] D. T. Son and N. Yamamoto, Phys. Rev. D 87, 085016 (2013).

[13] J.-W. Chen, S. Pu, Q. Wang, and X.-N. Wang, Phys. Rev. Lett. 110, 262301 (2013).

[14] C. Manuel and J. M. Torres-Rincon, Phys. Rev. D 89, 096002 (2014).

[15] J.-Y. Chen, D. T. Son, M. A. Stephanov, H.-U. Yee, and Y. Yin, Phys. Rev. Lett. 113, 182302 (2014).

[16] J.-Y. Chen, D. T. Son, and M. A. Stephanov, Phys. Rev. Lett. 115, 021601 (2015).

[17] Y. Hidaka, S. Pu, and D.-L. Yang, Phys. Rev. D 95, 091901 (2017).

[18] Y. Hidaka, S. Pu, and D.-L. Yang, Phys. Rev. D 97, 016004 (2018).

[19] Y. Hidaka and D.-L. Yang, Phys. Rev. D 98, 016012 (2018).

[20] N. Mueller and R. Venugopalan, Phys. Rev. D 96, 016023 (2017).
[21] N. Mueller and R. Venugopalan, Phys. Rev. D 97, 051901 (2018).

[22] A. Huang, S. Shi, Y. Jiang, J. Liao, and P. Zhuang, Phys. Rev. D 98, 036010 (2018).

[23] S. Carignano, C. Manuel, and J. M. Torres-Rincon, Phys. Rev. D 98, 076005 (2018).

[24] Ö. F. Dayi and E. Kilinçarslan, Phys. Rev. D 98, 081701 (2018).

[25] Y.-C. Liu, L.-L. Gao, K. Mameda, and X.-G. Huang, Phys. Rev. D 99, 085014 (2019).

[26] S. Lin and A. Shukla, J. High Energy Phys. 06 (2019) 060.

[27] U. W. Heinz, Phys. Rev. Lett. 51, 351 (1983).

[28] H. T. Elze, M. Gyulassy, and D. Vasak, Phys. Lett. B 177, 402 (1986).

[29] D. Vasak, M. Gyulassy, and H. T. Elze, Ann. Phys. (N.Y.) 173, 462 (1987).

[30] J.-P. Blaizot and E. Iancu, Phys. Rep. 359, 355 (2002).

[31] N. Mueller and R. Venugopalan, Phys. Rev. D 99, 056003 (2019).

[32] L. Adamczyk et al. (STAR Collaboration), Nature (London) 548, 62 (2017).

[33] J. Adam et al. (STAR Collaboration), Phys. Rev. C 98, 014910 (2018).

[34] Z.-T. Liang and X.-N. Wang, Phys. Rev. Lett. 94, 102301 (2005); 96, 039901(E) (2006).

[35] F. Becattini, L. Csernai, and D. J. Wang, Phys. Rev. C 88, 034905 (2013); 93, 069901(E) (2016).

[36] F. Becattini, V. Chandra, L. Del Zanna, and E. Grossi, Ann. Phys. (Amsterdam) 338, 32 (2013).

[37] R.-h. Fang, L.-g. Pang, Q. Wang, and X.-n. Wang, Phys. Rev. C 94, 024904 (2016).

[38] W. Florkowski, B. Friman, A. Jaiswal, and E. Speranza, Phys. Rev. C 97, 041901 (2018).

[39] D.-L. Yang, Phys. Rev. D 98, 076019 (2018).

[40] K. Fukushima, S. Pu, and Z. Qiu, Phys. Rev. A 99, 032105 (2019).

[41] W. Florkowski and R. Ryblewski, Prog. Part. Nucl. Phys. 108, 103709 (2019). 
[42] W. Florkowski, A. Kumar, and R. Ryblewski, Phys. Rev. C 98, 044906 (2018).

[43] K. Hattori, M. Hongo, X.-G. Huang, M. Matsuo, and H. Taya, Phys. Lett. B 795, 100 (2019).

[44] E. Gorbar, V. Miransky, I. Shovkovy, and X. Wang, Phys. Rev. D 88, 025025 (2013).

[45] M. Buzzegoli, E. Grossi, and F. Becattini, J. High Energy Phys. 10 (2017) 091; 07 (2018) 119(E).

[46] S. Lin and L. Yang, Phys. Rev. D 98, 114022 (2018).

[47] Y. Akamatsu and N. Yamamoto, Phys. Rev. Lett. 111, 052002 (2013).

[48] A. Ohnishi and N. Yamamoto, arXiv:1402.4760.

[49] D. Grabowska, D. B. Kaplan, and S. Reddy, Phys. Rev. D 91, 085035 (2015).

[50] N. Weickgenannt, X.-1. Sheng, E. Speranza, Q. Wang, and D. H. Rischke, Phys. Rev. D 100, 056018 (2019).

[51] J.-H. Gao and Z.-T. Liang, Phys. Rev. D 100, 056021 (2019).

[52] S. Ochs and U.W. Heinz, Ann. Phys. (N.Y.) 266, 351 (1998).
[53] V. Bargmann, L. Michel, and V. L. Telegdi, Phys. Rev. Lett. 2, 435 (1959).

[54] As in the massless case [17], the $\mathcal{O}\left(\hbar^{1}\right)$ terms proportional to $q^{\mu} \delta\left(q^{2}-m^{2}\right)$ can be absorbed into the distribution functions.

[55] M. Peskin and D. Schroeder, An Introduction to Quantum Field Theory (Westview Press, Boulder, CO, 1995).

[56] To be more precise, for both positive and negative $q \cdot n$, we should write $2 S_{m(n)}^{\mu \nu}=\bar{\epsilon}(q \cdot n) \epsilon^{\mu \nu \alpha \beta} q_{\alpha} n_{\beta} /(|q \cdot n|+m)$, where $\bar{\epsilon}(q \cdot n)$ denotes the sign for $q \cdot n$.

[57] More precisely, one has to include both fermions and antifermions and put an overall sign function $\bar{\epsilon}(q \cdot n)$ in front of the Wigner function.

[58] G. Prokhorov and O. Teryaev, Phys. Rev. D 97, 076013 (2018).

[59] G. Prokhorov, O. Teryaev, and V. Zakharov, Phys. Rev. D 98, 071901 (2018).

[60] K. Fukushima, D. E. Kharzeev, and H. J. Warringa, Nucl. Phys. A836, 311 (2010).

[61] K. Fukushima, Lect. Notes Phys. 871, 241 (2013). 